\title{
Mouse Retinal Cell Atlas: Molecular Identification of over Sixty Amacrine Cell Types
}

\author{
${ }^{\circledR}$ Wenjun Yan, ${ }^{1 \star}$ Mallory A. Laboulaye, ${ }^{1 *}{ }^{\circledR}$ Nicholas M. Tran, ${ }^{1 \star}$ Irene E. Whitney, ${ }^{1}{ }^{\oplus}$ Inbal Benhar, ${ }^{2}$ and \\ Doshua R. Sanes ${ }^{1}$ \\ ${ }^{1}$ Center for Brain Science and Department of Molecular and Cellular Biology, Harvard University, Cambridge, Massachusetts 02138, and ${ }^{2}$ Klarman \\ Cell Observatory, Broad Institute of MIT and Harvard, Cambridge, Massachusetts 02142
}

Amacrine cells (ACs) are a diverse class of interneurons that modulate input from photoreceptors to retinal ganglion cells (RGCs), rendering each RGC type selectively sensitive to particular visual features, which are then relayed to the brain. While many AC types have been identified morphologically and physiologically, they have not been comprehensively classified or molecularly characterized. We used high-throughput single-cell RNA sequencing to profile $>32,000$ ACs from mice of both sexes and applied computational methods to identify 63 AC types. We identified molecular markers for each type and used them to characterize the morphology of multiple types. We show that they include nearly all previously known AC types as well as many that had not been described. Consistent with previous studies, most of the AC types expressed markers for the canonical inhibitory neurotransmitters GABA or glycine, but several expressed neither or both. In addition, many expressed one or more neuropeptides, and two expressed glutamatergic markers. We also explored transcriptomic relationships among AC types and identified transcription factors expressed by individual or multiple closely related types. Noteworthy among these were Meis 2 and Tcf4, expressed by most GABAergic and most glycinergic types, respectively. Together, these results provide a foundation for developmental and functional studies of ACs, as well as means for genetically accessing them. Along with previous molecular, physiological, and morphologic analyses, they establish the existence of at least 130 neuronal types and nearly 140 cell types in the mouse retina.

Key words: GABA; glycine; Meis2; neuropeptide; RNA-seq; TCF4

\section{Significance Statement}

The mouse retina is a leading model for analyzing the development, structure, function, and pathology of neural circuits. A complete molecular atlas of retinal cell types provides an important foundation for these studies. We used high-throughput single-cell RNA sequencing to characterize the most heterogeneous class of retinal interneurons, amacrine cells, identifying 63 distinct types. The atlas includes types identified previously as well as many novel types. We provide evidence for the use of multiple neurotransmitters and neuropeptides, and identify transcription factors expressed by groups of closely related types. Combining these results with those obtained previously, we proposed that the mouse retina contains $\sim 130$ neuronal types and is therefore comparable in complexity to other regions of the brain.

Received Feb. 27, 2020; revised May 7, 2020; accepted May 13, 2020.

Author contributions: W.Y., M.A.L., N.M.T., I.E.W., and J.R.S. designed research; W.Y., M.A.L., N.M.T., and

I.E.W. performed research; I.B. contributed unpublished reagents/analytic tools; W.Y., M.A.L., N.M.T., and J.R.S. analyzed data; W.Y., M.A.L., N.M.T., and J.R.S. wrote the paper.

*W.Y., M.A.L., and N.M.T. contributed equally to this work.

This work was supported by the National Institutes of Health (Grants NS029169, MH105960, and K99EY029360); and the Human Frontier Science Program. We thank Drs. Thomas Bourgeron (Institut Pasteur, Paris, France), Isabelle Cloez-Tayarani (Institut Pasteur), and James Y. H. Li (University of Connecticut, Storrs, (T) for providing some mouse tissue used in this study; and Drs. Julia Kaltschmidt (Stanford University, Palo Alto, CA), Louis Reichardt (Simons Foundation, New York), Chinfei Chen (Harvard Medical School, Boston, MA), and Lisa Goodrich (Harvard Medical school) for providing mice.

The authors declare no competing financial interests.

Correspondence should be addressed to Joshua R. Sanes at sanesj@mcb.harvard.edu.

https://doi.org/10.1523/JNEUROSCI.0471-20.2020

Copyright $\odot 2020$ the authors

\section{Introduction}

The mouse retina is an experimentally tractable system for analyzing principles of CNS circuit development, structure, and function (Sanes and Masland, 2015; Seabrook et al., 2017). In addition, it is a prominent animal model for assessing mechanisms underlying retinal diseases, the major cause of irreversible blindness. An atlas of mouse retinal cell types would be a valuable resource for pursuing such studies. High-throughput singlecell RNA sequencing ( $\mathrm{scRNA}-\mathrm{seq}$ ) is a promising method for achieving this goal. It enables comprehensive identification and molecular characterization of the cell types that comprise complex tissues, as well as a framework for incorporating structural and physiological data required for generating a definitive atlas (Zeng and Sanes, 2017). Moreover, it provides molecular 
markers that facilitate the development of genetic strategies to access and manipulate specific cell types within neural circuits.

In an initial study, we used scRNA-seq to profile $\sim 45,000$ cells from mouse retinas, recovering the six major classes of cells present in vertebrate retinas: photoreceptors (PRs) that sense light; three classes of interneurons [horizontal cells (HCs), bipolar cells (BCs), and amacrine cells (ACs)] that receive and process information from PRs; retinal ganglion cells (RGCs) that receive information from interneurons and transmit it to central targets; and Müller glial cells (Macosko et al., 2015; Fig. 1A). This study was unable, however, to resolve all of the cell types into which the classes are divided: only 33 neuronal groups were recovered, although the number of authentic types had been estimated to exceed 60 (Masland, 2012). The reason was that $\sim 80 \%$ of retinal cells are rod PRs (Jeon et al., 1998), leaving the less abundant but more heterogeneous classes undersampled (i.e., BCs, ACs, and RGCs), precluding the recovery of rare types or resolution of types with similar gene expression profiles. Accordingly, we set out to enrich BCs, RGCs, and ACs so we could profile them in sufficient numbers. For BCs and RGCs, we documented the existence of 15 and 46 types, respectively (Shekhar et al., 2016; Tran et al., 2019). These numbers correspond well to those obtained from recent high-throughput physiological, ultrastructural, and molecular studies (Baden et al., 2016; Greene et al., 2016; Franke et al., 2017; Bae et al., 2018; Rheaume et al., 2018).

Here, we present an analysis of ACs. ACs receive synaptic input from BCs and other ACs, and provide output to BCs, other ACs, and RGCs. They modify the visual signals that travel from photoreceptors to RGCs via BCs, thereby shaping the visual features to which each RGC type responds. Several AC types have been shown to play specific roles in retinal computation; for example, some render RGCs selectively responsive to motion in particular directions, and others capable of distinguishing local from global motion (Werblin, 2010; Vaney et al., 2012; Krishnaswamy et al., 2015; Chen and Guestrin, 2016; Lee et al., 2016; Diamond, 2017; Wei, 2018). These diverse roles require multiple AC types; indeed, they are generally thought to be the most heterogeneous retinal class (MacNeil and Masland, 1998; Lin and Masland, 2006; Fig. 1B).

Our transcriptomic analysis revealed 63 AC types, enabling us to identify markers for all and characterize morphology for many of them. Because ACs are known to display remarkable heterogeneity in neurotransmitter phenotype, we systematically analyzed the expression of neurotransmitter biosynthetic enzymes and neuropeptide precursors, providing evidence for the presence of at least 20 small-molecule or small-peptide transmitters in ACs, with potential use of multiple transmitters in the majority of them. We also analyzed transcriptional relationships among types and identified transcription factors expressed by closely related types. They include Meis2 and Tcf4, which are expressed by most GABAergic and glycinergic types, respectively.

Combined with results from other classes, our inventory of ACs provides what we believe to be a nearly complete mouse retinal cell atlas, comprising $\sim 140$ cell types. Thus, at least in this respect, the retina is about as complicated as any other part of the brain.

\section{Materials and Methods}

Animals. Animals were used in accordance with National Institutes of Health $(\mathrm{NIH})$ guidelines and protocols approved by the Institutional Animal Care and Use Committee (IACUC) at Harvard University. The following knock-in and transgenic mouse lines were obtained from The Jackson Laboratory: Chx10-cre-GFP [stock \#005105 (Chx10 is now named Vsx2); Rowan and Cepko, 2004]; Slc17a7-IRES2-Cre (stock \#023527; Harris et al., 2014); Cck-IRES-Cre (stock \#012706; Taniguchi et al., 2011); Penk-IRES2-Cre (generated at the Allen Institute; stock $\# 025112$ ); Sst-IRES-Cre (stock \#013044); and Thy1-mitoCFP-P (stock \#007967; Misgeld et al., 2007). Contactin 5-lacZ and Contactin 6-lacZ lines were from Li et al. (2003) and Takeda et al. (2003) via Julia Kaltschmidt (Stanford University, Palo Alto CA) and Thomas Bourgeron (Institut Pasteur, Paris, France), respectively. NeuroD6-cre knock-in mice (Goebbels et al., 2006) were obtained from K. Nave (Max Planck Institute, Göttingen, Germany) via L. Reichardt (UCSF, San Francisco CA). The Gbx2-CreERT2-IRES-GFP line (Chen et al., 2009) was a gift from James Y. H. Li (University of Connecticut School of Medicine) via the Chinfei Chen laboratory (Harvard Medical School). The Ptfla-cre (Kawaguchi et al., 2002) line was obtained from Lisa Goodrich (Harvard Medical School), The Thy1-STP-YFP Line 15 was generated in our laboratory (stock \#005630, The Jackson Laboratory; Buffelli et al., 2003). Some tissue was obtained from mice analyzed in a previous study (Martersteck et al., 2017). Ptf1a-cre was maintained on a CD1 background (stock \#022, Charles River Laboratories). All other mutants were maintained on a C57BL/6J background (stock \#000664, The Jackson Laboratory). Mice of both sexes were used interchangeably; we detected no obvious differences between sexes.

Cell sorting and single-cell sequencing. Postnatal day 19 (P19) Chx10cre-GFP animals were sacrificed by intraperitoneal injection of Euthasol. Eyes were removed and retinas dissected in oxygenated Ames solution between 10:00 and 11:00 A.M. Retinas were incubated at $37^{\circ} \mathrm{C}$ for $10 \mathrm{~min}$ in a papain solution followed by trituration in an ovomucoid solution to quench papain activity and generate a single-cell suspension. Cells were centrifuged at $450 \times g$ for $8 \mathrm{~min}$, and the pellet was resuspended in Ames solution plus 4\% BSA with eBioscience rat anti-mouse CD133-APC (Thermo Fisher Scientific) and eBioscience rat anti-mouse CD73-PE (Thermo Fisher Scientific). Following incubation for $15 \mathrm{~min}$ at room temperature, cells were washed with Ames solution plus BSA, centrifuged again, and resuspended at a concentration appropriate for flow cytometry. Samples were sorted on a MoFlo Astrios cell sorter (Beckman), and cells triple negative for GFP, APC, and PE, but positive for the cell viability marker Calcein Blue, were collected. They were then processed according to the 10x Genomics version 2 Chromium Single Cell 3' Reagent Kit (10x Genomics; Zheng et al., 2017). Briefly, single cells are partitioned into oil droplets containing single oligonucleotidederivatized beads followed by cell lysis, barcoded reverse transcription of RNA, amplification, shearing, and attachment of $5^{\prime}$ adaptor and sample index oligos. Libraries were sequenced on the Illumina HiSeq 2500 (paired end reads: read 1, $26 \mathrm{bp}$; read 2, $98 \mathrm{bp}$ ).

Adult amacrine cells were collected and sequenced as part of unrelated projects (Tran et al., 2019; I. Benhar, I.E. Whitney, N.M. Tran, and J.R. Sanes, unpublished observations).

Computational methods. We analyzed scRNA-seq data following the pipeline detailed in Peng et al. (2019). Briefly, sample demultiplexing was performed with the cellranger mkfastq function (version 2.1.0, Chromium, 10x Genomics), and reads were aligned to the reference genome mm10 version 1.2.0 from cellranger refdata using the cellranger count function with the option force - cells $=6000$. Clustering was performed to stratify cells into major classes using defining class-selective markers, and then to cluster amacrine cells into putative types. Subsequent steps were as follows (1) A threshold of 600 genes detected per cell was applied to filter out low-quality cells and debris. (2) A gene expression matrix was calculated as follows: The count matrix was first normalized by total number of unique molecular identifiers (UMIs) for each cell and multiplied by the median UMI count per group, then log transformed after adding 1 (Shekhar et al., 2016). (3) Highly variable genes were identified by the method of Pandey et al. (2018). (4) Batch correction was performed on the expression matrix of highly variable genes using a linear regression model in the Seurat package (https:// satijalab.org/seurat/). (5) Principal component (PC) analysis was applied, and significant PCs estimated based on Tracy-Widom theory (Patterson et al., 2006) were used for further analysis of clustering. (6) Data were partitioned into clusters of transcriptionally related cells using the Louvain algorithm with the Jaccard correction (Shekhar et al., 2016). (7) 
A

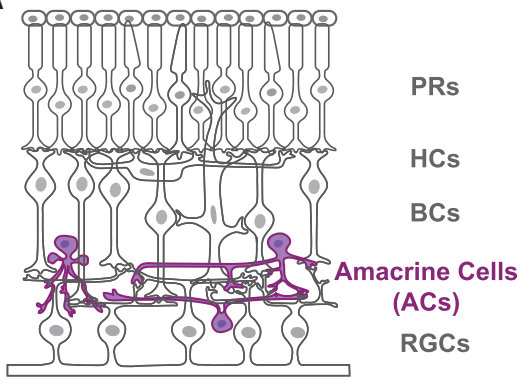

B

Morphological diversity of amacrine types

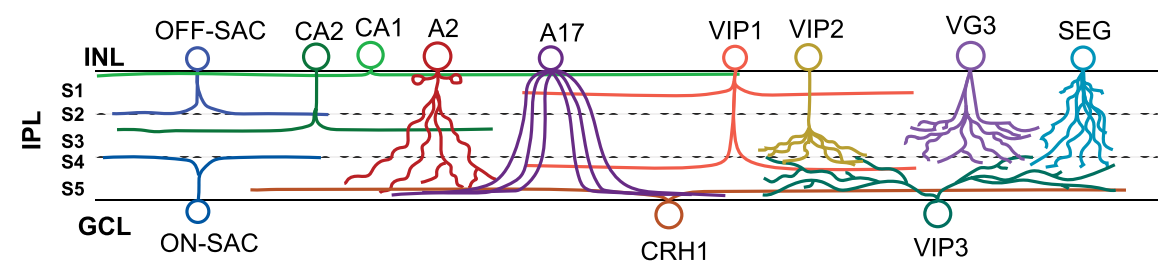

C

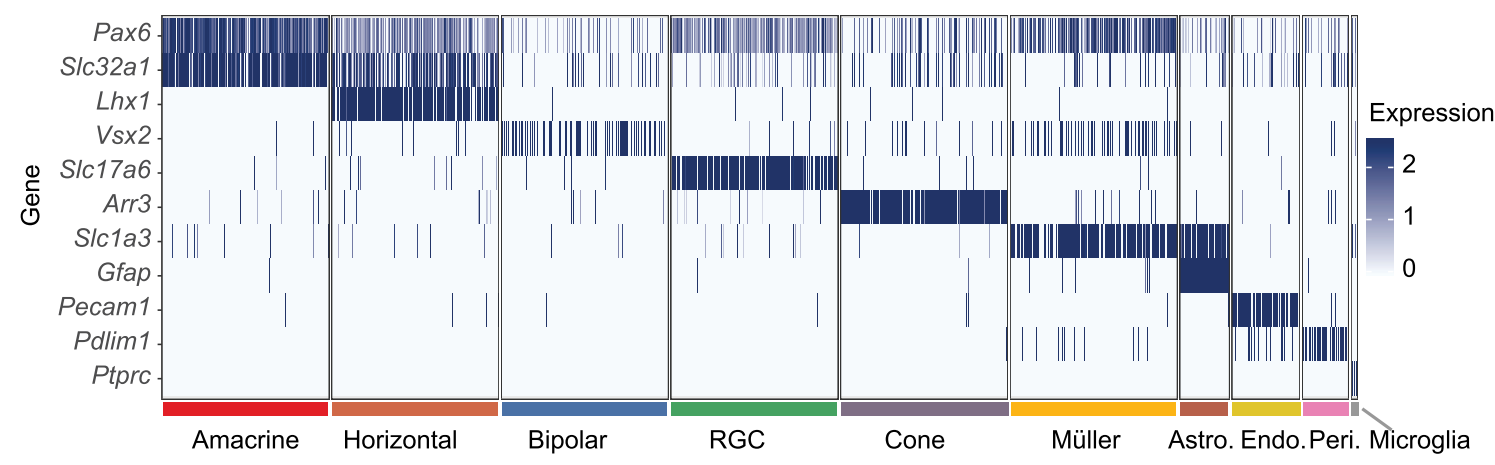

D

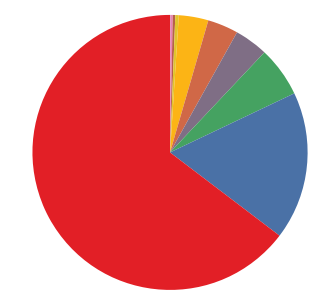

Class

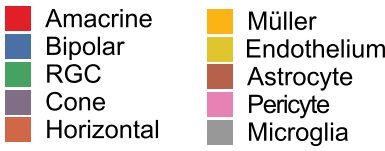

Horizontal Microglia
E

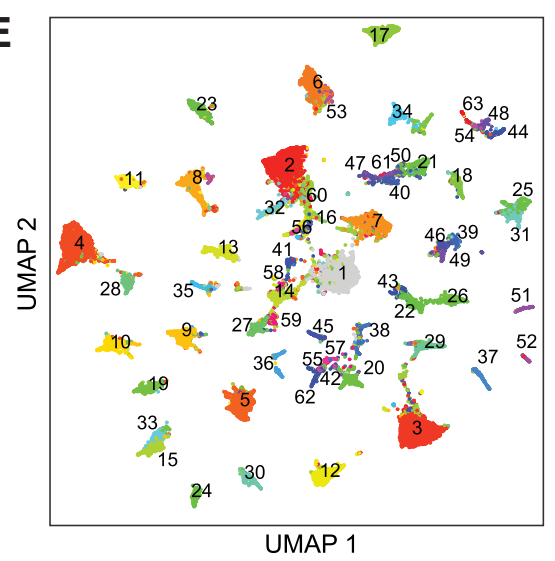

$\mathbf{F}$

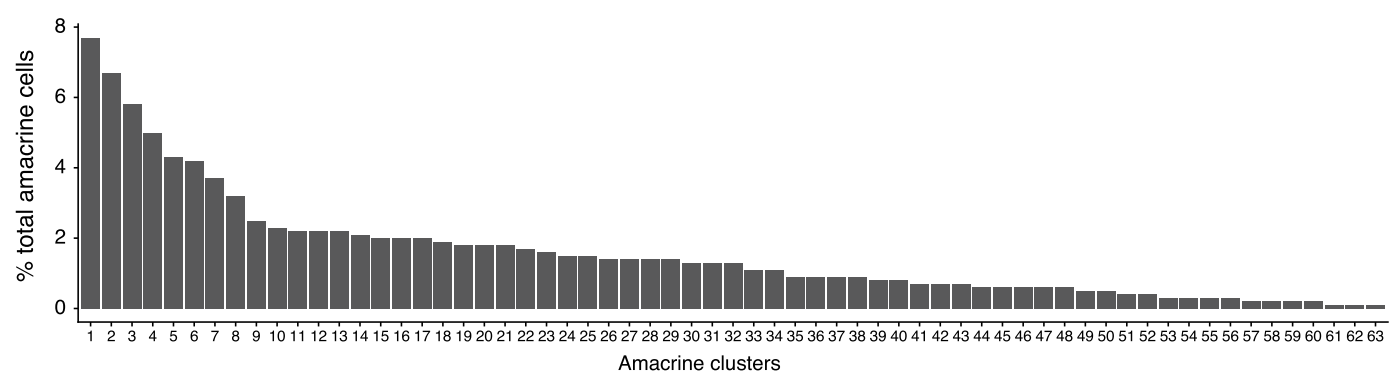

Figure 1. Single-cell transcriptomes of mouse amacrine cells. A, Sketch of a retinal cross section showing cell classes and layers (adapted from Tran et al., 2019). ACS (purple) are interneurons that synapse with rod bipolar cells, other ACs, and RGCS. B, ACs have diverse dendritic morphologies, as illustrated by the lamination patterns of previously defined AC types. INL, Inner nuclear layer; GCL, ganglion cell layer. IPL sublaminae represented as S1-5. C, Expression patterns of a subset of marker genes used to allocate retinal cells to classes. Plot shows transcript count per cell of a randomly downsampled subset of all cells. Color bars indicate cell class. Astro, Astrocyte; Endo, endothelium; Peri, pericyte. D, Fraction of cells in each cell class, as determined by the expression of canonical markers in $\boldsymbol{C}$. $\boldsymbol{E}$, UMAP visualization of $63 \mathrm{AC}$ clusters numbered in order of abundance. $\boldsymbol{F}$, Relative frequencies of AC clusters, expressed as a percentage of 32,523 AC cells profiled. Clusters are numbered in order of decreasing frequency.

A dendrogram was built on the expression matrix of highly variable genes for the assigned clusters to reveal their overall transcriptomic similarity. (8) Clusters closest to each other on the dendrogram were assessed for differential expression (DE), and merged if no more than six DE genes were found (log fold change $>1$; adjusted $p<0.001$ ). DE tests were performed using the R package MAST (Finak et al., 2015). (9) Because some contaminants (i.e., non-ACs or doublets) became evident only following clustering, we reexamined the clustered data to remove 
them. The criteria for doublets included having an increased number of transcripts per cell and having combined expression of canonical markers from different cell classes. (10) We retested for doublets among amacrine cells using the R package "DoubletFinder" (McGinnis, et al., 2019) with the default parameter of $7.5 \%$ as the expected doublet rate. We found that $\sim 55 \%$ of cells in clusters 16 , and 60 could be doublets, although we have no way of verifying this possibility. As a second test for amacrine-amacrine doublets, we asked whether each cluster expressed genes at far higher levels than any other cluster. Although there are some variations among clusters, C16 was suspect, but all others appeared to be authentic. (11) To compare types between datasets, we trained multiclass classifiers (Xgboost algorithm; Chen and Guestrin, 2016) using the R package "xgboost" and assigning matches as detailed in Peng et al. (2019) and van Zyl et al. (2020). (12) For visualization purposes only, dimensionality was further reduced to $2 \mathrm{D}$ using Uniform Manifold Approximation and Projection (UMAP).

Histology. Following euthanizing with Euthasol, mouse eyes were removed and fixed in $4 \%$ paraformaldehyde (PFA) in $1 \times$ PBS. In most cases, PFA/PBS was administered by transcardiac perfusion followed by eye removal and a $20 \mathrm{~min}$ post-fix in PFA/PBS. Alternatively, eyes were removed immediately and fixed in PFA/PBS for $90 \mathrm{~min}$ on ice. Eyes were then rinsed with PBS and the retina dissected out. Retinas to be sectioned were incubated in $30 \%$ sucrose in PBS overnight at $4^{\circ} \mathrm{C}$, after which they were embedded in tissue-freezing medium, frozen in dry ice, and stored at $-80^{\circ} \mathrm{C}$ until processing. Retinas were cryosectioned at 20$25 \mu \mathrm{m}$ and air dried. The sections were rehydrated in PBS, incubated in $5 \%$ normal donkey serum and $0.3 \%$ Triton X-100 in PBS for $1 \mathrm{~h}$, incubated with primary antibodies overnight at $4^{\circ} \mathrm{C}$, washed in PBS, incubated with secondary antibodies for $2 \mathrm{~h}$ at room temperature, washed again in PBS, allowed to dry, mounted with Vectashield (Vector Laboratories), and coverslipped.

For whole mounts, retinas were blocked in 5\% donkey serum, $0.3 \%$ Triton X-100 in PBS for 3-14 h and incubated in primary antibody for $5-7 \mathrm{~d}$ at $4^{\circ} \mathrm{C}$. Retinas were then washed in PBS and incubated overnight in secondary antibody. Finally, retinas were washed in PBS, flat mounted on cellulose membrane filters (Millipore), coverslipped with Fluoro-Gel (Electron Microscopy Sciences), and sealed with nail polish.

The following antibodies were used: rabbit and chicken anti-GFP (1:2000; Millipore/1:1000; catalog \#06-896, Abcam); goat anti-choline acetyltransferase (ChAT; 1:500; catalog \#AB144P, Millipore); goat anti-vesicular acetylcholine transporter (VAChT; 1:500; catalog \#sc-7717, Santa Cruz Biotechnology); rabbit anti-Tfap2b (1:200; catalog \#2509, Cell Signaling Technology); rabbit anti-Rbpms (1:500; catalog \#194213, Abcam); guinea pig anti-Rbpms (1:1000; catalog \#1832-RBPMS, PhosphoSolutions); goat anti-Vsx2 (1:300; catalog \#21690, Santa Cruz Biotechnology); rabbit antiVglut3 (1:1000; catalog \#135203, Synaptic Systems); rabbit anti-Ebf3 (1:2000; catalog \#AB10525, Millipore); rat anti-CD140a-PE (1:100; catalog \#135905, Thermo Fisher Scientific); rabbit anti-Nfix (1:1000; catalog \#PA530897, Thermo Fisher Scientific); rat anti-somatostatin (1:500; catalog \#MAB354, Millipore); rabbit anti- $\beta$-GAL (1:5000; in-house); rabbit antiPpp1r17 (1:1000; catalog \#HPA047819, Atlas Antibodies); rabbit antiNeuropeptide Y (1:1000; catalog \#10980, Abcam); rabbit anti-GHRH (1:500; catalog \#ab187512, Abcam); goat anti-Glyt1 (1:10,000; catalog \#AB1770, Chemicon); mouse anti-GAD 65/67 [1:500; catalog \#GAD-6, Developmental Studies Hybridoma Bank (DSHB)]; rabbit anti-GAD65/67 (1:1000; catalog \#AB1511, Millipore); mouse anti-Pax6 (1:500; catalog \#Pax6-s, DSHB); mouse anti-Meis2 (1:100; catalog \#1A11, DSHB); guinea pig anti-Prdm8 (1:2000; gift from the Sarah E. Ross laboratory, University of Pittsburgh, Pittsburgh, PA); guinea pig anti-Lhx9 \#1342 (1:5000; gift from the Jane Dodd laboratory, Columbia University Medical Center, New York, NY); mouse anti-Tfap2c (1:500; catalog \#a3108, Sigma-Aldrich); rabbit anti-Calbindin (1:2000; catalog \#CB38a, Swant); mouse anti-Calretinin (1:5000; catalog \#MAB1568, Millipore); rabbit anti-Neurod2 (1:500; catalog \#ab104430, Abcam); rabbit anti-Tbr2 (1:500; catalog \#ab183991, Abcam); and rabbit anti-TCF4 (1:200; catalog \#22337-1-AP, Proteintech). Secondary antibodies against various species were conjugated to Alexa Fluor 488 (Invitrogen), Alexa Fluor 568 (Invitrogen), or Alexa Fluor 647 (Jackson ImmunoResearch) and used at 1:1000. Nuclei were stained with ToPro Cy5 (1:5000, Thermo Fisher Scientific).
To sparsely label ACs, we injected mice from Cre-expressing lines listed above with a cre-dependent virus, AAV9-EF1a-BbTagBY (Cai et al., 2013; catalog \#45185-AAV9, Addgene), AAV9-EF1a-BbChT (Cai et al., 2013; catalog \#45186-AAV9, Addgene), or AAV9-CAG-tdTomato (catalog \#51503-AAV9, Addgene). Intravitreal injections were performed as described in the study by Tran et al. (2019). Animals were killed 3-4 weeks after injection, and retinas were processed as described above.

Probe generation for in situ hybridization. To generate probes, RNA was extracted from P19 C57BL/6J mouse retina and reverse transcribed as described in the study by Laboulaye et al. (2018). Probes were generated from cDNA by PCR using Q5 Polymerase and the following sets of primers: Car3 (901 bp): forward, 5'-ATCTTCACTGGGGCTCCTCT-3'; reverse: $5^{\prime}$-gaaattaatacgactcactatagggCGCATACTCCTCCATACCCG-3'; Kit (1017 bp): forward: 5' -TGGTCAAAGGAAATGCACGA-3'; reverse: $5^{\prime}$-gaaattaatacgactcactatagggTCTTCTTAGCGTGACCAG-3'; and Slc17a7 (1028 bp): forward: 5' -CGGATACTCGCACTCCAAGG-3'; reverse: 5' gaaattaatacgactcactatagggTTCCCTCAGAAACGCTGGTG-3'.

PCR products were separated by gel electrophoresis, after which bands of the expected size were column purified and sequenced. Confirmed PCR templates were then transcribed by T7 RNA polymerase (Roche) with a digoxigenin (DIG)-UTP nucleotide mix (Roche). The resulting products were precipitated overnight at $-80^{\circ} \mathrm{C}$ in mixture of $\mathrm{TE}$ buffer, $\mathrm{LiCl}$, and $\mathrm{EtOH}$. The following day, samples were centrifuged. Pellets were then washed with $70 \% \mathrm{EtOH}$ and resuspended with 1:1 formamide/water. Finally, probes were run on agarose gel to ensure expected size.

Fluorescence in situ hybridization. Tissue was collected, prepared with RNase-free reagents and sectioned as described above. Slides were processed as described in the study by Tran et al. (2019). Briefly, slides were fixed in $4 \%$ PFA for $10 \mathrm{~min}$ and then rinsed $2 \times 5 \mathrm{~min}$ PBS with $0.1 \%$ Tween-20 (PBT). Slides were then digested in a Proteinase-K solution of $0.5 \mu \mathrm{g} / \mathrm{ml}$ for $3 \mathrm{~min}$, washed $2 \times 5 \mathrm{~min}$ in PBT, and fixed again in $4 \%$ PFA for $5 \mathrm{~min}$. Slides were then washed $2 \times 5 \mathrm{~min}$ in PBT, incubated in acetylation solution ( $0.1 \mathrm{M}$ triethanolamine $+0.25 \%$ acetic anhydride) for $5 \mathrm{~min}$. Slides were then washed $2 \times 5 \mathrm{~min}$ in PBT and incubated in prehybridization solution for $1 \mathrm{~h}$ at room temperature. DIG-labeled probes were denatured for $5 \mathrm{~min}$ at $85^{\circ} \mathrm{C}$ and added to slides at 1:200 dilution. Slides were then coverslipped and incubated overnight at $65^{\circ} \mathrm{C}$.

On the second day, slides were washed $2 \times$ in prehybridization solution, $2 \times$ in $2 \times$ saline sodium citrate (SSC), as follows: $2 \times$ in $2 \times$ SSC, and $2 \times$ in $0.2 \times$ SSC. All of these washes were conducted for $30 \mathrm{~min}$ each at $65^{\circ} \mathrm{C}$. Slides were then washed $2 \times$ in maleic acid buffer containing $0.1 \%$ Tween-20 (MABT) at room temperature and blocked in heatinactivated sheep serum/MABT/blocking solution for $1 \mathrm{~h}$. Slides were then incubated overnight with anti-DIG-HRP antibodies (1:750).

On the third day, slides were washed $6 \times 5 \mathrm{~min}$ in MABT, followed by $2 \times 5 \mathrm{~min}$ in PBT. Signals were amplified with Cy3-tyramide (1:200) for $1 \mathrm{~h}$ (TSA-Plus System; Perkin-Elmer Life Sciences). Slides were rinsed $6 \times 5 \mathrm{~min}$ in PBT and $2 \times 5 \mathrm{~min}$ in $1 \times$ PBS and then incubated in $3 \%$ donkey serum and $0.3 \%$ Triton-X in $1 \times$ PBS for $30 \mathrm{~min}$, followed by primary antibody incubation overnight at $4^{\circ} \mathrm{C}$.

On the last day, slides were washed $3 \times 5 \mathrm{~min}$ in $1 \times$ PBS. Slides were then incubated in secondary antibodies for $2 \mathrm{~h}$ at room temperature, washed $3 \times 5 \mathrm{~min}$ in $1 \times \mathrm{PBS}$, dried, coverslipped, and sealed.

Images were acquired on an Olympus-FV1000 Confocal Microscope. We used Image (NIH) software to analyze confocal stacks and generate maximum intensity projections.

Experimental design and statistical analysis. Statistical methods for analysis of RNA-seq data are detailed above (see Computational methods). To quantify immunostaining combinations (see Fig. 9), confocal images were analyzed in Image); four or more images from two or more retinas were analyzed for each marker combination. Custom ImageJ macros were used to place circular regions of interest (ROIs; diameter, $3.44 \mu \mathrm{m}$ ) over all cell somas and nuclei that were positive for at least one marker. Fluorescent intensity was measured for each marker in each ROI in single Z-slice images. Fluorescent values for ROIs from each marker were minimum subtracted and normalized to the maximum value for plotting. 

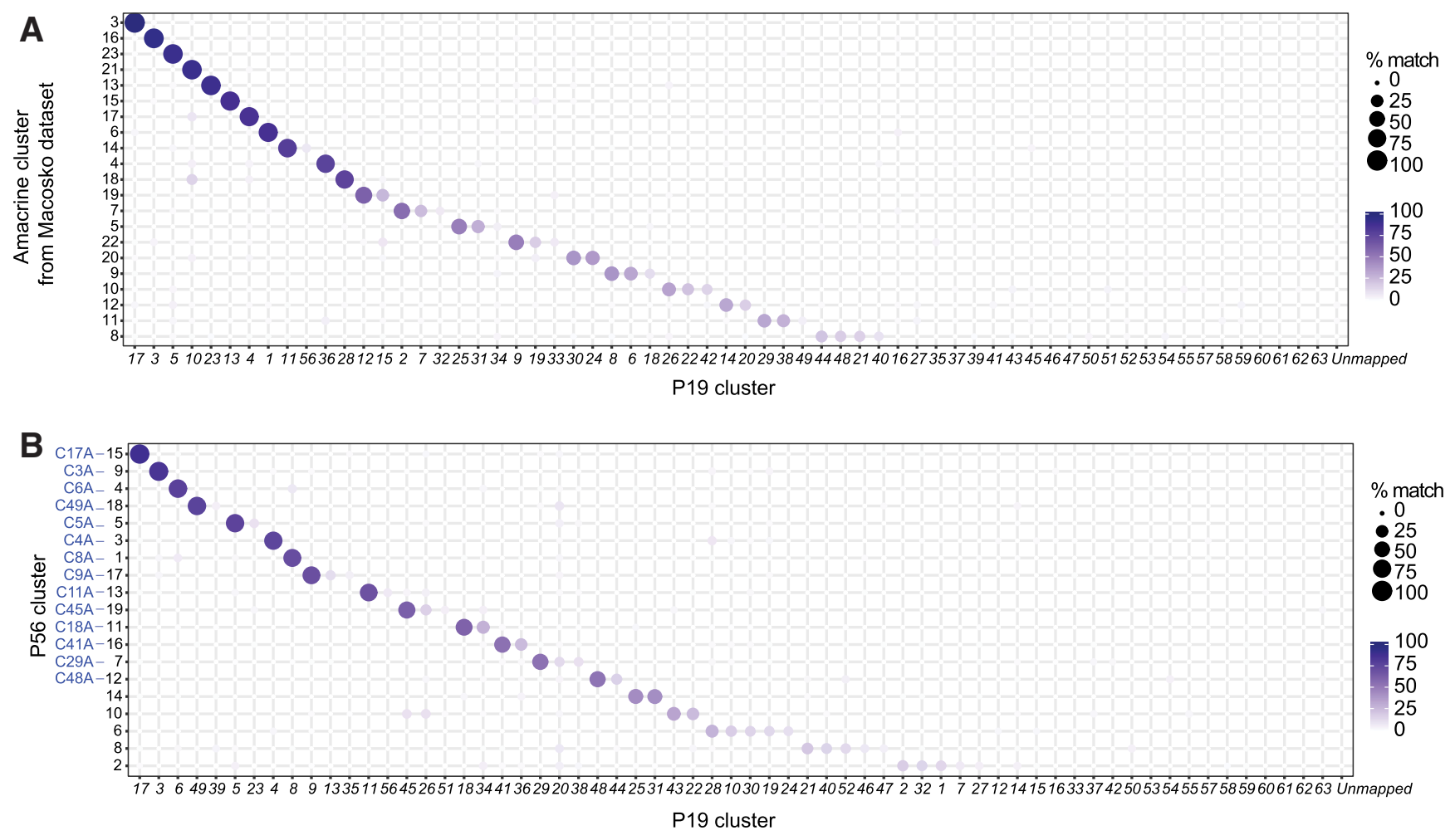

Figure 2. No additional AC types were detected in two other datasets. A, Comparison of AC clusters using cells obtained with selective depletion of other cell classes (this study) or without selection (Macosko et al., 2015). Transcriptional correspondence is depicted as a matrix in which circle diameter and color indicate the percentage of cells in a given AC type from the new data $(x$-axis) that are assigned to a particular type from Macosko et al. (2015; $y$-axis) by a classification algorithm (xgBoost) trained on the P19 data. For the $y$-axis, clusters were ordered by the degree of match to a single P19 cluster. While some AC types were not represented in the study by Macosko et al. (2015), due to lower sampling, all types detected by Macosko et al. (2015) were represented in the new dataset, suggesting selective depletion did not remove any AC types. B, Comparison of AC clusters obtained in this study with those sampled from P56 retina. While some AC types were not represented in the undersampled P56 data, all P56 types were represented in the new dataset. We renamed P56 clusters in which $>50 \%$ cells mapped to single P19 clusters with their P19 counterparts' cluster ID plus "A."

Data availability. Ram and processed datasets reported in this study are available through the Gene Expression Omnibus with accession number GSE149715. The single-cell data can be visualized in the Broad Institute Single Cell Portal at https://singlecell.broadinstitute.org/single_ cell/study/SCP919.

\section{Results}

Transcriptomic separation of amacrine cells into 63 clusters In previous studies aimed at generating $\mathrm{BC}$ and RGC atlases, we enriched cells using class-specific transgenic markers, Vsx2/ Chx10 and VGlut2, respectively (Shekhar et al., 2016; Tran et al., 2019). We were unable to find a suitable class-specific marker for ACs and therefore adopted a strategy of selective depletion. We dissociated retinas from P18 to P19 Vsx2-GFP mice, in which BCs and Müller glia are labeled (Rowan and Cepko, 2004; Shekhar et al., 2016), and labeled rod and cone photoreceptors with fluorophore-conjugated antibodies to CD73 and CD133, respectively (Lakowski et al., 2011; Peng et al., 2019). We isolated GFP/CD73/CD133 triple-negative cells by FACS and submitted them to droplet-based scRNA-seq (Zheng et al., 2017), obtaining high-quality transcriptomes from 55,287 cells. We then divided the cells into classes by the expression of canonical markers (Peng et al., 2019; Fig. 1C). ACs were defined as cells that were positive for the transcription factor $\operatorname{Pax} 6$ and the vesicular inhibitory amino acid transporter Slc32a1, and negative for markers of other classes; they comprised $58.8 \%$ of the population (Fig. 1D) or a total of 32,523 ACs. Unsupervised analysis divided the ACs into 63 clusters, each being a putative cell type
(Fig. 1E). They ranged in abundance from $0.1 \%$ to $7.7 \%$ of all ACs (Fig. 1F). Since ACs comprise $7-10 \%$ of retinal cells (Jeon et al., 1998; Macosko et al., 2015), individual types comprise $\sim 0.01-1 \%$ of all retinal cells.

Before proceeding further, we performed three tests to assess the possibility that our collection protocol had excluded AC types. First, we immunostained "uncollected" cells from the FACS isolation procedure with the AC marker TFAP2B, and found negligible numbers of positive cells, indicating that few if any $\mathrm{AC}$ types were $\mathrm{GFP}^{+}, \mathrm{CD}^{+} 3^{+}$, or $\mathrm{CD}_{133^{+}}$(data not shown). Second, we compared our types to the 21 groups identified from a collection that included no enrichment or depletion steps (Macosko et al., 2015). We used a classifier based on a supervised machine-learning algorithm, Xgboost (Chen and Guestrin, 2016). As expected, in many cases, a single cluster in the smaller dataset ( 4,400 ACs) mapped to multiple clusters in the current dataset, indicating improved resolution in distinguishing cell types. Importantly, however, no clusters from the Macosko et al. (2015) dataset were left unmatched in our data (Fig. 2A). Finally, we asked whether some types might emerge later than P19. To this end, we queried a set of 5347 ACs collected from P56 mice in the course of unrelated studies (Tran et al., 2019; I. Benhar, I.E. Whitney, N.M. Tran, and J.R. Sanes, unpublished observations). As with the AC data from the study by Macosko et al. (2015), the P56 AC data were underpowered to resolve every type but were sufficient to resolve 20 clusters. Again, all P56 AC clusters mapped to one or more P19 clusters, and in no case was a P56 cluster unmatched in the P19 dataset, as might occur if further diversification occurred following P19 (Fig. 2B). Together, 
these results support the idea that our dataset includes most if not all AC types that comprise $>0.01 \%$ of retinal cells.

\section{Correspondence between clusters and AC types}

To match molecularly defined clusters to authentic AC types, we began by identifying DE genes for each cluster. Most clusters could be uniquely identified by the expression of a single DE gene (Fig. $3 A$ ), and the others by a combination of two DE genes. Expression patterns of established type-specific markers allowed assignment of several clusters to known AC types (Table 1). They included starburst ACs (SACs; C17: Chat; Vaney et al., 2012); AII ACs (C3: Gjd2, Prox1, Dab1, Nfia, Dner; Rice and Curran, 2000; Hansen et al., 2005; Pérez de Sevilla Müller et al., 2017; Keeley and Reese, 2018); SEGs [C4: Satb2, Ebf3, and Glyt1 (Slc6a9); Kay et al., 2011]; VG3 ACs [C13: VGlut3 (Slc17a8); Haverkamp and Wässle, 2004; Johnson et al., 2004; Grimes et al., 2011; Krishnaswamy et al., 2015; Lee et al., 2016; Tien et al., 2016]; and A17 ACs [C6: Prkca (PKC $\alpha), S d k 1, \mathrm{Calb2}^{-}, \mathrm{Dab1}^{-}$; Grimes et al., 2010; Puthussery and Fletcher, 2007; Yamagata and Sanes, 2018]. Other types expressed neuropeptides known to mark specific AC types; they include Cck (C10, C17, C18, C34; Firth et al., 2002), Vip (C22, C26, C47; Akrouh and Kerschensteiner, 2015; Park et al., 2015, 2018; Pérez de Sevilla Müller et al., 2019), Crh (C37; Zhu et al., 2014; Jacoby et al., 2015), and Penk (C35, C59, C63; Chen et al., 2013). For some of these types, we were able to validate new markers, such as Car3 for VG3 cells (Fig. 3B,C). Thus, our atlas includes most if not all previously characterized AC types.

To characterize AC types that had not, to our knowledge, been studied previously, we combined fluorescence in situ hybridization or immunohistochemistry with sparse labeling to reveal cellular morphology. We used lines that express Cre recombinase in subsets of ACs, and infected retinas with adenoassociated viral (AAV) vectors that express a fluorescent protein in a Cre-dependent manner. For example, immunostaining sections from an AAV-infected Ptfla-cre line, which results in broad expression in newly born ACs (Fujitani et al., 2006), showed that $\mathrm{C} 23\left(\mathrm{Cd}_{\left.140 a^{+}\right)}\right.$is a medium-field AC type that stratifies in the center of the inner plexiform layer (IPL; Fig. 3D, $E$ ). Additional examples are presented below. Thus, although our mapping of molecular to morphologic types is not exhaustive, we expect that most or all of the AC clusters identified in our dataset correspond to authentic cell types.

As a further test of these assignments, we examined clusters from the adult (P56) dataset that matched with a single one of the 63 AC clusters. For convenience, we renumbered these clusters so that they correspond to their P19 counterpart (Fig. 2B), referring to P56 cluster 9 as C3A, P56 cluster 3 as C4A, and so on. They included starburst, A17, SEG, and AII ACs as well as Nos $1^{+}$and $\mathrm{Cck}^{+}$clusters. In each case, most or all of the defining markers identified at P19 were retained at P56 (Fig. 4). A notable exception was a catecholaminergic type (C45, C45A), which is discussed below.

\section{Neurotransmitters and neuromodulators}

Most ACs are inhibitory neurons that use GABA or glycine as neurotransmitters. However, it has long been known that subsets of ACs also contain a variety of other small-molecule and small-peptide neurotransmitters and neuromodulators (Karten and Brecha, 1983). Our AC atlas provided an opportunity to systematically characterize these subsets. For this analysis, we used hierarchical clustering to arrange the types by transcriptomic similarity (Fig. 5A), so we could assess relationships among types that shared transmitters.

\section{GABA and glycine}

We examined the expression of the GABA synthetic enzyme glutamate decarboxylase ( $\mathrm{Gad} 1$ and $\mathrm{Gad} 2$ ), and GABA transporters Gat 1-3 (Slc6a1, Slc6a13, and Slc6a11) as markers of GABAergic cells; and the expression of glycine transporters GlyT1 and GlyT2 (Slc6a9 and Slc6a5) as markers of glycinergic cells (Fig. 5A). Most informative were the canonical markers Gad1, Gad2, and Glyt1. As expected, the expression of Gad $(1+2)$ and GlyT1 was mutually exclusive in most types (56 of 63), with 43 of the 63 types being GABAergic and 13 being glycinergic (Fig. $5 A, B$ ). The GABAergic and glycinergic types were entirely restricted to different clades with the exception of SACs (C17), which were distinct from either clade (see Discussion).

The remaining seven types had unconventional neurotransmitter expression patterns (Fig. 5B). Four types (C10, C24, C30, and C36) expressed low levels of both GABAergic and glycinergic markers, and likely represent "non-GABAergic, non-glycinergic" (nGnG) ACs (Kay et al., 2011; Macosko et al., 2015); further analysis of these types is presented below.

The three remaining types (C16, C53, and C62) expressed high levels of both $\mathrm{Gad}(1+2)$ and GlyT1, raising the possibility that they use both transmitters. We asked whether clusters might be composed of "doublets," arising from co-occupancy of a single microbead by two cells. This may indeed be the case for C16, as judged by a doublet-detecting algorithm, but is unlikely for the others (see Materials and Methods). Moreover, we observed a sparse population of ACs that was positive for both GAD and GlyT1 by immunostaining (Fig. $5 C$ ).

Of the four nGnG types, three were members of the glycinergic clade, while the fourth was a member of the GABAergic clade. The putative $\mathrm{GABA}^{+}$glycine ${ }^{+}$types were all members of the GABAergic clade. GABAergic and glycinergic types include both abundant and rare types (Fig. $1 F$ ), whereas the dual and $n G n G$ types were all rare ( $<2 \%$ of ACs per type). Overall, the GABAergic, glycinergic, $n G n G$, and dual ACs comprise $\sim 67 \%$, $25 \%, 6 \%$, and $2 \%$, respectively, of all ACs.

\section{Glutamate}

One well studied AC type, VG3, expresses VGlut3 as well as Glyt1, and is capable of both excitatory glutamatergic and inhibitory glycinergic transmission, likely at different synapses (Lee et al., 2016; Tien et al., 2016). We assessed the expression of all three vesicular glutamate transporters. VGlut1 (Slc17a7), VGlut2 (Slc17a6), and VGlut3 (Slc17a8; Fig. 5D). VGlut3 expression was confined to the VG3 type and VGlut2, an RGC marker, was not detectably expressed by any AC type.

Surprisingly, we found one rare type, C56 ( $0.3 \%$ of all ACs), that expressed VGlut1, implying the existence of a second glutamatergic AC type. These cells, which we call VG1 ACs, also expressed GABAergic markers and could potentially mediate dual excitatory glutamatergic and inhibitory GABAergic transmission. We labeled these cells using a VGlut1-Cre line crossed to a Cre-dependent reporter (Thy1-STP-YFP; Buffelli et al., 2003). In addition to bipolar cells, all of which are VGlut $1^{+}$, and a rare VGlut ${ }^{+}$RGC type (Tran et al., 2019), we detected VGlut $^{+}$cells that were ACs by the criteria that they were positive for the AC marker TFAP2B and negative for the bipolar marker VSX2 (Fig. 5E,F). VG1 ACs laminated in S1 of the IPL (we divide the IPL into five sublaminae, with S1 abutting the inner nuclear layer and S5 abutting the ganglion cell layer) and have narrowly stratified but widely ramifying dendrites, which, consistent with their expression of $\mathrm{Gad}$, is more characteristic of GABAergic (wide-field) than glycinergic (narrow-field) ACs. 


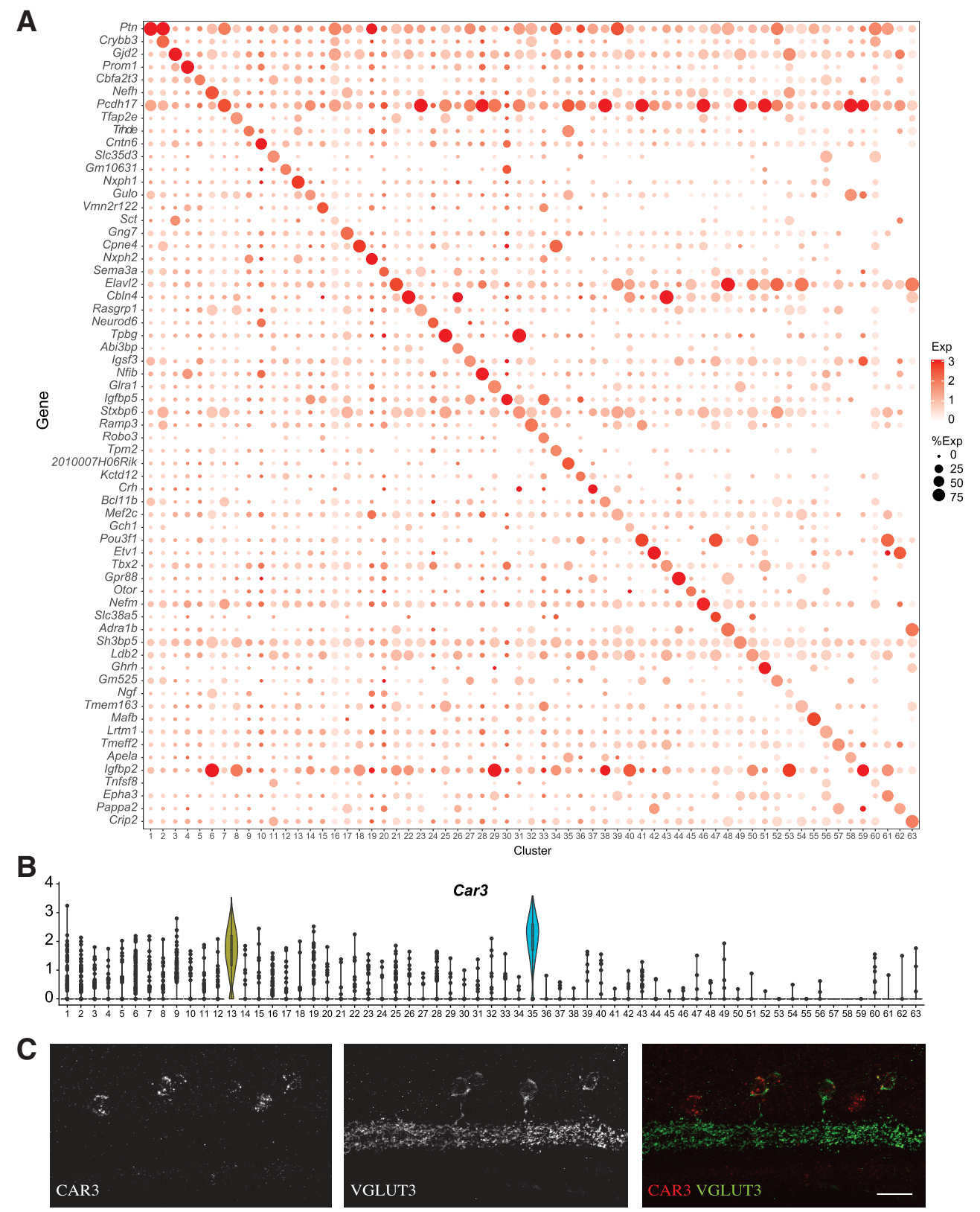

D

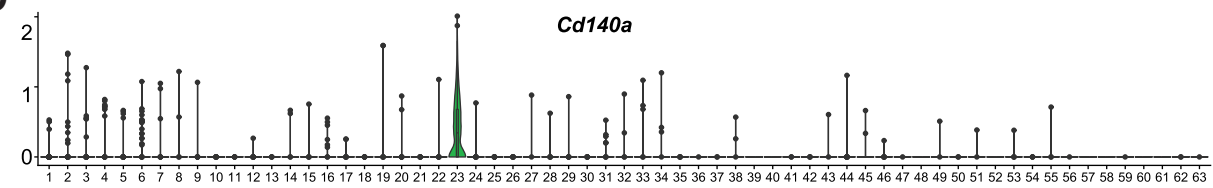

E

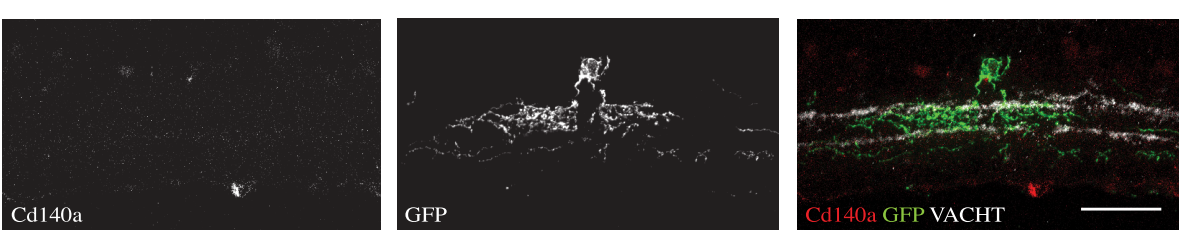

Figure 3. Molecular markers of $\mathrm{AC}$ types. $A$, Dot plots showing genes (rows) that uniquely mark $\mathrm{AC}$ clusters (columns). In this and subsequent dot plots, the size of each circle is proportional to the percentage of cells expressing the gene, and the color depicts the average transcript count in expressing cells, unless otherwise indicated. $\boldsymbol{B}$, Violin plots representing the expression of Car3 in all AC types. Expression is highest in C35 (VG3 ACs) but is also substantial in C13. Violins are drawn for clusters in which transcripts were detected in $>20 \%$ of cells. For clusters below this threshold, the transcript levels for Car3-expressing cells are shown by dots. C, Retinal section doubly labeled for Car3 (in situ hybridization) and VGlut3 (immunohistochemistry). VG3 ACs are Car3 ${ }^{+}$. Scale bar, $20 \mu \mathrm{m}$. D, Violin plot showing selective expression of Cd140a in C23. E, Retinal section sparely labeled with GFP (Ptf1-cre $\times$ Cre-dependent AAV) and CD140A antibody reveals the morphology of C23 ACs. Scale bar, $40 \mu$ m. 
Table 1. Summary of AC types

\begin{tabular}{|c|c|c|c|}
\hline Cluster & GABAergic/glycinergic & Cells profiled $(n)$ & Type or key marker \\
\hline 1 & GABAergic & 2505 & \\
\hline 2 & GABAergic & 2190 & \\
\hline 3 & Glycinergic & 1897 & All \\
\hline 4 & Glycinergic & 1618 & SEG \\
\hline 5 & GABAergic & 1396 & \\
\hline 6 & GABAergic & 1353 & A17 \\
\hline 7 & GABAergic & 1216 & \\
\hline 8 & GABAergic & 1029 & \\
\hline 9 & Glycinergic & 800 & \\
\hline 10 & Neither & 754 & $\mathrm{nGnG-2;CCK}$ \\
\hline 11 & GABAergic & 726 & \\
\hline 12 & Glycinergic & 721 & \\
\hline 13 & Glycinergic & 707 & VG3 \\
\hline 14 & GABAergic & 698 & \\
\hline 15 & Glycinergic & 663 & \\
\hline 16 & Both & 638 & \\
\hline 17 & GABAergic & 637 & SAC \\
\hline 18 & GABAergic & 621 & CCK \\
\hline 19 & Glycinergic & 588 & \\
\hline 20 & GABAergic & 585 & \\
\hline 21 & GABAergic & 574 & \\
\hline 22 & GABAergic & 559 & VIP \\
\hline 23 & GABAergic & 533 & \\
\hline 24 & Neither & 480 & $n G n G-1$ \\
\hline 25 & GABAergic & 480 & CA-II \\
\hline 26 & GABAergic & 470 & VIP \\
\hline 27 & GABAergic & 468 & \\
\hline 28 & Glycinergic & 462 & \\
\hline 29 & GABAergic & 459 & \\
\hline 30 & Neither & 435 & $n G n G-3$ \\
\hline 31 & GABAergic & 433 & \\
\hline 32 & GABAergic & 421 & \\
\hline 33 & Glycinergic & 360 & \\
\hline 34 & GABAergic & 348 & CCK \\
\hline 35 & Glycinergic & 300 & PENK \\
\hline 36 & Neither & 289 & $n G n G-4$ \\
\hline 37 & GABAergic & 280 & CRH \\
\hline 38 & GABAergic & 277 & \\
\hline 39 & GABAergic & 262 & \\
\hline 40 & GABAergic & 257 & \\
\hline 41 & GABAergic & 230 & \\
\hline 42 & GABAergic & 221 & \\
\hline 43 & GABAergic & 220 & \\
\hline 44 & GABAergic & 210 & \\
\hline 45 & GABAergic & 206 & CA-I \\
\hline 46 & GABAergic & 203 & \\
\hline 47 & GABAergic & 197 & VIP \\
\hline 48 & GABAergic & 184 & nNOS \\
\hline 49 & GABAergic & 175 & \\
\hline 50 & GABAergic & 159 & \\
\hline 51 & GABAergic & 130 & GHRH \\
\hline 52 & GABAergic & 118 & nNOS \\
\hline 53 & Both & 109 & \\
\hline 54 & GABAergic & 91 & nNOS \\
\hline 55 & GABAergic & 89 & \\
\hline 56 & GABAergic & 86 & VG1 \\
\hline 57 & GABAergic & 81 & \\
\hline 58 & GABAergic & 81 & \\
\hline 59 & GABAergic & 77 & PENK \\
\hline 60 & GABAergic & 64 & \\
\hline 61 & GABAergic & 41 & \\
\hline 62 & Both & 34 & \\
\hline 63 & GABAergic & 28 & PENK, SST \\
\hline
\end{tabular}

Acetylcholine

Starburst ACs (C17, Chat $\left.^{+}\right)$are intensively studied cholinergic ACs. We assessed the expression of Chat, the VAChT Slc18a3, and the high-affinity choline transporter Slc5a7. All were expressed selectively by $\mathrm{C} 17$ (Fig. $5 G$ ), supporting the idea that starburst ACs are the only cholinergic ACs. ON and OFF starburst ACs are molecularly distinct in neonates (Peng et al., 2020), but differences between these closely related subtypes were no longer detectable by P19.

\section{Monoamines}

Monoamine neurotransmitters include dopamine, norepinephrine, epinephrine, serotonin, tyramine, tryptamine, and histamine. We assessed the expression of their synthetic enzymes (Th, Ddc, Pnmt, Dbh, Tph1, Tph2; Fig. 5H). Tyrosine hydroxylase (TH), which generates DOPA from tyrosine, was expressed at high levels in C25, a GABAergic type, and at lower levels in several other groups, consistent with evidence for at least two dopaminergic AC types, which vary in TH levels (Zhang et al., 2007; Vuong et al., 2015).

Surprisingly however, the other enzyme required for synthesis of dopamine, DOPA decarboxylase (Ddc; generates dopamine from DOPA) was not detectably expressed in C25, but traces of expression were detected in three clusters with low levels of $\mathrm{TH}$ (C4, C10, and C45). To understand this result, we examined the expression of monoamine synthetic enzymes in adult ACs and found heterogeneity between clusters expressing $\mathrm{TH}$ (Fig. 4H). C45A expressed the highest levels of both $\mathrm{TH}$ and Ddc, marking it as the CAI AC. This cluster also selectively expressed connexin 45 (Gja1), which has been reported to label cells with a morphology characteristic of CAI ACs (Theofilas et al., 2017). C25A expressed lower levels of TH and no detectable Ddc, which is consistent with it being the CAII AC, in which dopamine and $\mathrm{TH}$ have been difficult to detect (Vuong et al., 2015). Additional molecular markers that may serve to differentiate between CAI and CAII ACs include Chl1 and Arhgdig. Other enzymes involved in the synthesis of monoamine neurotransmitters were not expressed at significant levels by ACs at either P19 or P56 (Fig. 5; data not shown).

\section{Gasotransmitters}

Three gases have been implicated as neurotransmitters: nitric oxide (NO), carbon monoxide (CO), and hydrogen sulfide (H2S; Boehning and Snyder, 2003). Three AC types, C48, C52, and C54, expressed high or moderate levels of nitric oxide synthase (Nos1; Fig. 5I), consistent with previous reports of at least two $\mathrm{Nos}^{+}$AC types (Pang et al., 2010; Zhu et al., 2014; Jacoby et al., 2018). Heme oxygenases (Hmox1, Hmox2) generate CO, with Hmox 2 thought to be responsible for generating $\mathrm{CO}$ used as a neurotransmitter. Hmox 2 was broadly expressed by ACs (Fig. $5 H)$, whereas cystathionine $\gamma$-lyase $(\mathrm{Cth})$ and cystathionine $\beta$-synthase $(\mathrm{Cbs})$, which synthesize $\mathrm{H} 2 \mathrm{~S}$, were not expressed at high levels by any ACs. Thus, we find no evidence for selective synthesis of $\mathrm{CO}$ or $\mathrm{H} 2 \mathrm{~S}$ by specific AC types.

\section{Neuropeptides}

We next mapped expression of genes encoding neuropeptides, which support a wide range of neuromodulatory and signaling roles (Fig. 6A). In addition to those mentioned above, they included Neuropeptide Y $(N p y)$, cocaine- and amphetamineregulated transcript (Cartpt), tachykinin (Tac1), somatostatin $(S s t)$, B-endorphin $(P o m c)$, and galanin $(\mathrm{Gal})$. Neuropeptides were expressed by ACs from all small-molecule neurotransmitter 


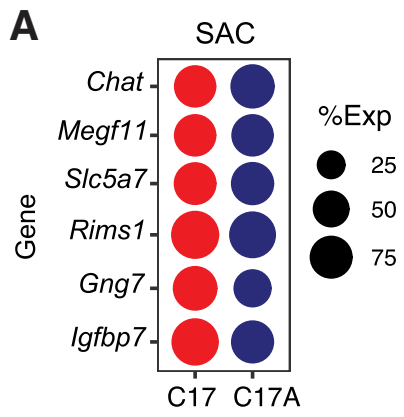

E

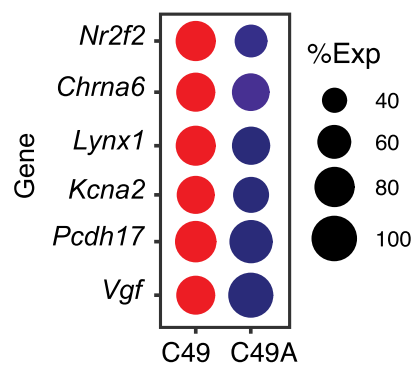

B

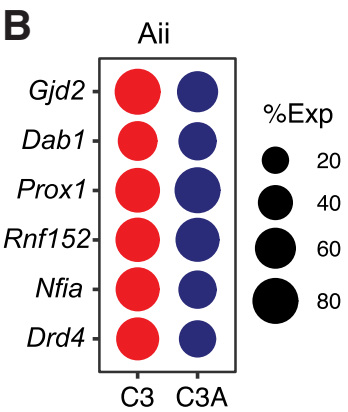

$\mathbf{F}$

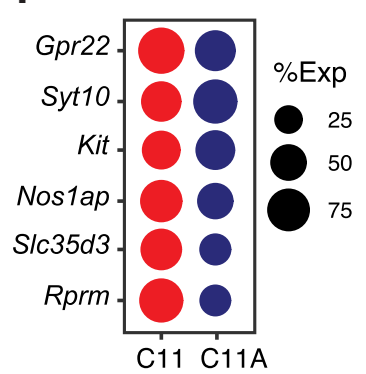

C

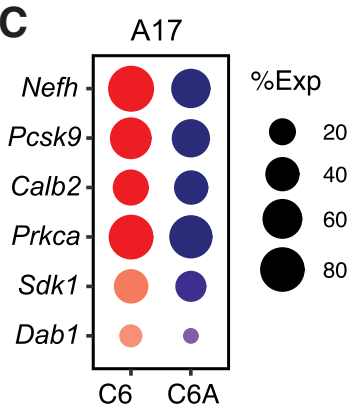

G

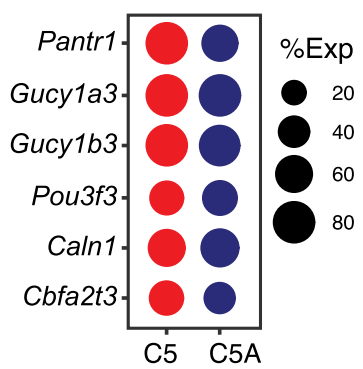

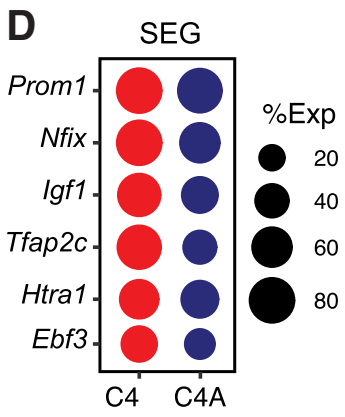

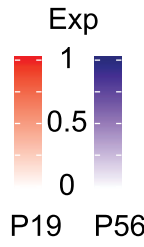

H

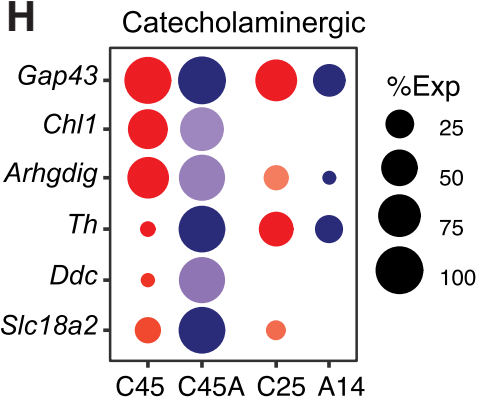

Figure 4. Expression of cell type-specific genes in selected types of ACs at P19 and in adults. $\boldsymbol{A}-\boldsymbol{H}$, Dot plots show marker gene expression in P19 and P56 ACs for Starburst amacrine cells

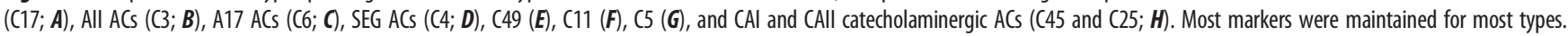
For catecholaminergic ACS, however, levels of dopamine synthetic enzymes (Th, Ddc) and the monoamine transporter (Slc18a2) were several-fold higher in adults than at P19 in C45, suggesting that C45 is the CAI AC type. Adult cluster A14 is the closest match to the P19 C25 (Fig. 2B).

classes. We also observed the expression of several neuromodulators previously studied in gut, brain, and other tissues, but not, to our knowledge, in retina, including angiotensin, calcitonin, somatomedin, and ghrelin. In contrast, we observed no significant expression of 45 other peptides (Fig. $6 F$ ).

Several neuropeptides were expressed by multiple AC types. For one of them, proenkephalin (encoded by the Penk gene), we combined immunohistochemistry with sparse labeling to assess the morphology of individual types. Penk is expressed at the highest levels by C35, C59, and C63 (Fig. 6B). These types were distinguished by selective expression of Ppp1r17 and Car3 (C35), or Sst and Gal (C63); C59 expresses none of these genes. We marked and characterized Penk $^{+}$cells by injecting a Cre-dependent AAV reporter into a Penk-Cre mouse line. C35 ACs $\left(\mathrm{Ppp} 1 \mathrm{r17}^{+}\right)$are narrow-field ACs; C59 ACs (Sst- and Ppp1r17-) are medium-field ACs; and C63 ACs (Sst $\left.{ }^{+}\right)$are wide-field ACs with dendrites in multiple sublaminae, including S1, S3, and S5 (Fig. 6C-E). Because of the density of labeling, we were unable to determine whether individual ACs are multistratified or whether processes of individual C63 ACs are confined to a single sublamina.

\section{nGnG amacrines}

As noted above, four AC types expressed substantially lower levels of GABAergic and glycinergic markers than known GABAergic or glycinergic types (Fig. $5 A, B$ ). We call these types nGnG-1-4. To characterize these types, we identified additional markers that distinguished them from each other and from other ACs (Fig. 7A). We used these markers to characterize each nGnG type using immunohistochemistry and a set of transgenic lines.

nGnG-1 ACs (C24) expressed Neurod6, Ebf3, and Ppp1r17, which we previously showed to define the $\mathrm{nGnG}$ ACs labeled in the MitoP mouse line (Kay et al., 2011, Macosko et al., 2015). Consistent with previous results, it was labeled in a Neurod6-Cre line, as detected by immunostaining for Cre recombinase, coexpressed PPP1R17, and was negative for NFIX (Fig. 7B,C).

nGnG-2 ACs (C10) expressed Cntn6, Cck, Ebf3, Nfix, and Prdm8. We labeled them in Cntn6-lacZ and Cck-IRES-Cre lines, and confirmed their nGnG status by immunostaining for GAD using an antibody that recognizes both GAD65 and GAD67 (encoded by Gad1 and Gad2) and GLYT1. We found that these cells were negative for GAD, GlyT1. and PPP1R17, but were positive for NFIX and EBF3 (Fig. 7D-J).

nGnG-3 ACs (C30) expressed Cntn5 and Ppp1r17. We labeled them in a Cntn5-LacZ line and showed that they coexpressed PPP1R17 (Fig. 7K). The density of Cntn5-LacZ labeling in bipolar cells and RGCs precluded further examination of this cell type.

nGnG-4 (C36) expressed Gbx2 and Lhx9. We labeled them in a Gbx2-Creer-GFP line and confirmed that both populations were $\mathrm{GAD}^{-}$and GLYT1 ${ }^{-}$, which is further supported by an independent study (Kerstein et al., 2020) Their somata were present in both the inner nuclear layer and ganglion cell layer. We additionally observed coexpression of LHX9 by immunostaining (Fig. $7 L-N)$.

Using a combination of transgenic mouse lines and immunohistochemical markers, we were able to document the morphology of three of the $\mathrm{nGnG}$ types. $\mathrm{nGnG}-1$ and $\mathrm{nGnG}-2$ were narrow-field types (similar to glycinergic ACs, to which they are related; see above) with dendrites that arborize in S1-3 and S1-4, respectively (Fig. 7O,P). nGnG-4 ACs had arbors tightly confined to S3 and appeared to be medium-field or wide-field ACs, similar to GABAergic ACs, to which they are related; Fig. 7Q). The lamination pattern of these ACs is similar to that of CAII ACs (Vuong et al., 2015), which we believe correspond to C25, but they do not express detectable levels of TH or other catecholaminergic markers.

Interestingly, three of the $\mathrm{nGnG}$ types (nGnG1-3) expressed $\operatorname{Lgr5}$ (Fig. 7A), a gene that has been studied intensively as a 
A

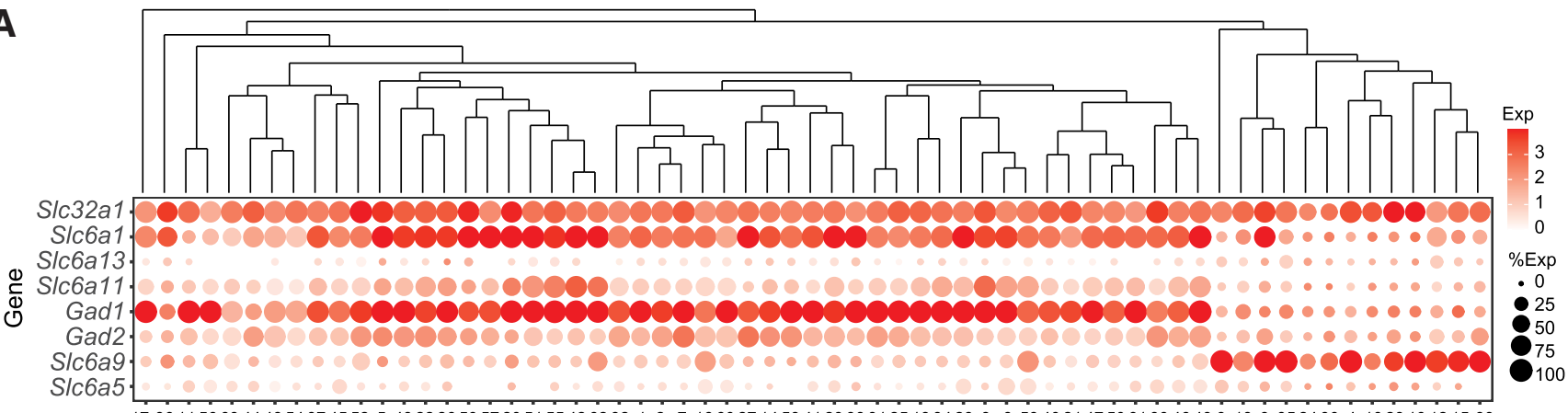

173611566344485437455254322265957205155426232127166027145841293831251834238653402147506139464931393524304102819121533 Cluster

B

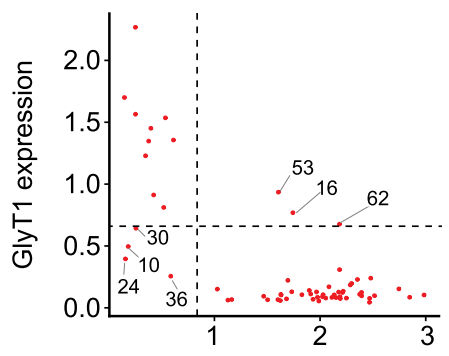

Glutamate Decarboxylase expression
C

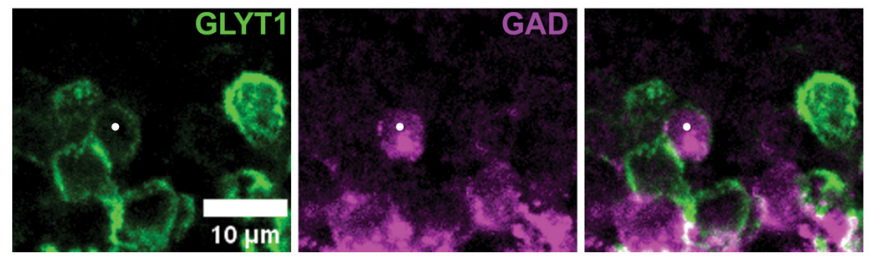

D

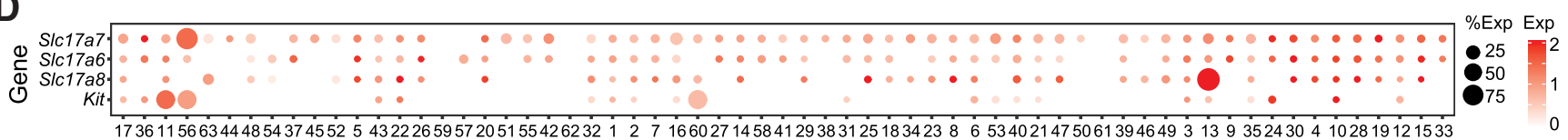
Cluster

$\mathbf{E}$
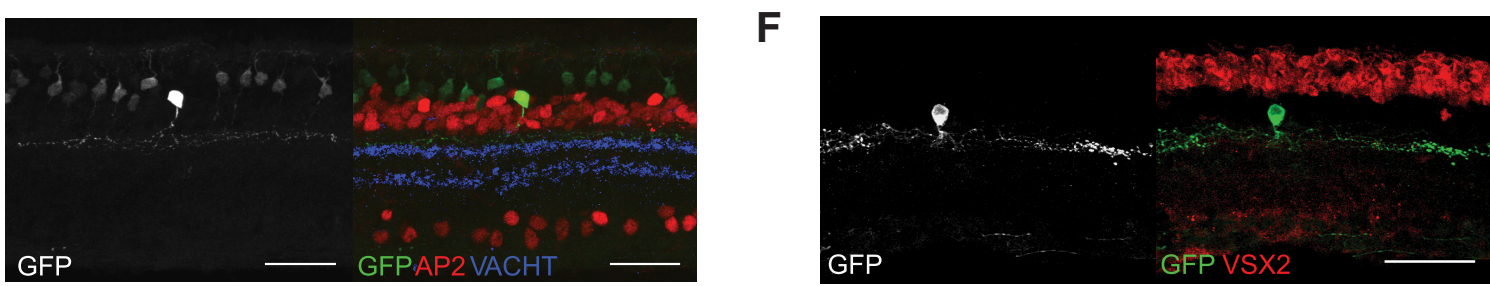

G

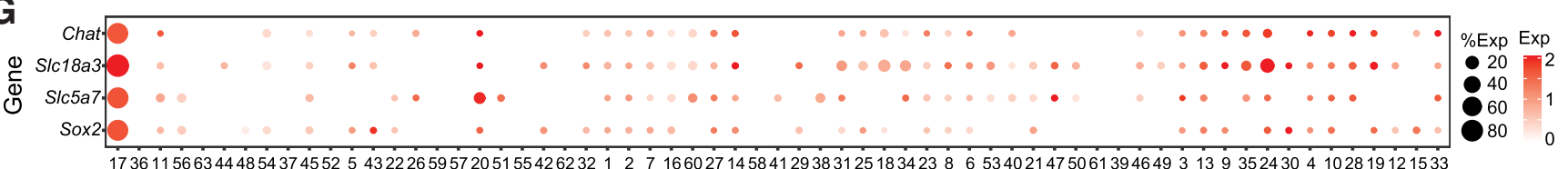

Cluster

H

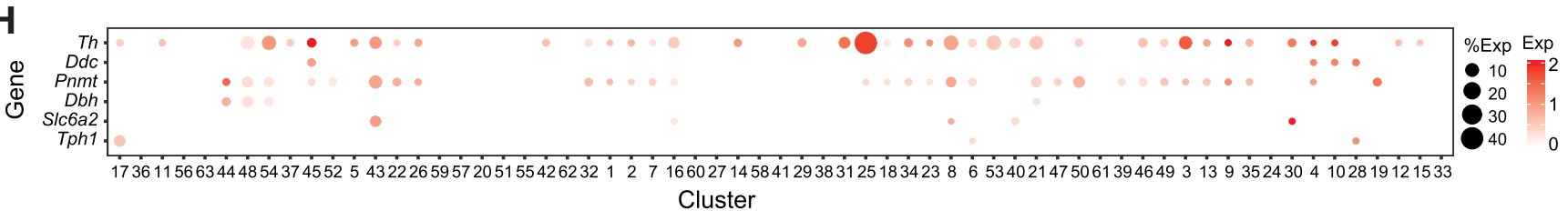

I

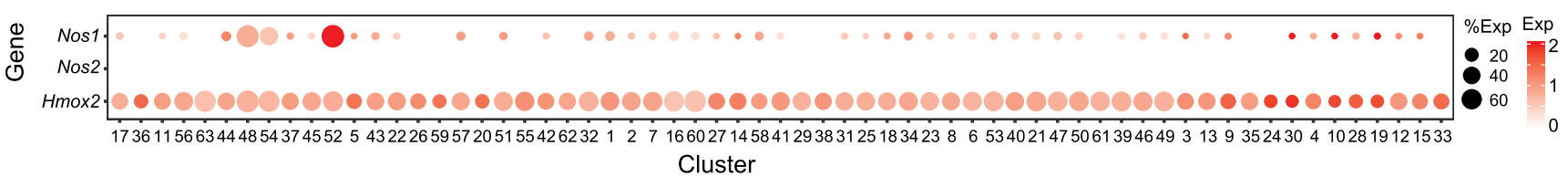

Figure 5. Evidence for multiple small molecule transmitters in ACs. A, Expression of GABAergic and glycinergic markers in ACs. SIc6a1, SIc6a13, and S/c6a11 encode GABA transporter types 1, 2, and 3, respectively. Gad1 and Gad2 encode two forms of glutamate decarboxylase. S/c6a9 and S/c6a5 encode vesicular glycine transporters 1 and 2, respectively. B, Expression of the most informative GABAergic (Gad1 and Gad2) and glycinergic (Slc6a9) markers divides ACs into the following four groups: GABAergic (43 types), glycinergic (11 types), neither (nGnGs, 4 types), and potentially dual GABAergic and glycinergic (3 types). C, Immunostaining of P22 retinas revealed sparsely populated ACs (white dot) that coexpress GABAergic (GAD65/67) and glycinergic (GLYT1) markers. Scale bar, $10 \mu \mathrm{m}$. D, Distinct AC clusters express glutamate transporters Slc17a7 (VGlut1; VG1 ACs) and S/c17a8 (VGlut3; VG3 ACs); none express S/c17a6 (VGlut2). E, $\boldsymbol{F}$, Characterization of VG1 ACs, labeled in 15 VGlut1-Cre $\times$ Thy1-STP-YFP line mice and detected by immunostaining with $\alpha$-GFP antibody. VG1 ACS are AP2 ${ }^{+}$(D) and VSX2 ${ }^{-}(\boldsymbol{E})$. Scale bars, 
marker of stem and progenitor cells in multiple tissues, and is a critical regulator their activity (Leung et al., 2018). It was recently reported that a set of $\mathrm{Lgr}^{+} \mathrm{ACs}$ are able to re-enter the cell cycle and generate new retinal neurons and glia (Chen et al., 2015). These might be nGnG ACs, consistent with their low level of canonical GABAergic and glycinergic AC markers. Lgr5 is also expressed by two close transcriptional relatives of these $\mathrm{nGnG}$ ACs, C4 (SEGs) and C28, both of which are glycinergic; this expression is consistent with a report that many $\operatorname{Lgr} 5^{+}$ACs are glycinergic (Sukhdeo et al., 2014).

\section{Receptors for neurotransmitters and neuromodulators}

ACs form synapses on BCs, RGCs, and other ACs. We asked whether these cells express receptors for the transmitters and modulators that ACs might use. To this end, we queried data from 46 RGC types (Tran et al., 2019) and 15 BC types (Shekhar et al., 2016), as well as data from ACs generated in this study (Figs. 8, 9).

In general, receptors were broadly expressed. All $\mathrm{BC}, \mathrm{AC}$, and RGC types expressed at least some GABA, glycine, and glutamate receptor subunits, consistent with the large number of GABAergic, glycinergic, and glutamatergic neurons that form synapses in the inner plexiform layer. Perhaps more surprising, many acetylcholine, dopamine, and neuropeptide receptors were broadly expressed, although only a few cell types use these transmitters or modulators, and their synapses are confined to a few sublaminae within the inner plexiform layer. For example, dopamine synthetic enzymes were expressed at high levels in two amacrine clusters, but dopamine receptors were found in multiple types of ACs, BCs, and RGCs. Likewise, although there is only a single cholinergic retinal cell type (SACs), both nicotinic and muscarinic acetylcholine receptors were expressed broadly.

Some receptor subunits were, however, selectively expressed by a small number of cell types. They include the glutamate receptor subunits Grial, Grik1, and Grin $3 a$; glycine receptor Glyra1; GABA receptor subunits Gabrd, Gabrb2, and Gabra1; dopamine receptors $\operatorname{Drd} 2, \operatorname{Drd} 4$, and $\operatorname{Drd5}$; cholinergic receptors Chrna3, Chrna6, and Chrm2; and neuropeptide receptors Sstr2 and Npy1r. Interestingly, some adrenergic and serotonin receptors were also selectively expressed, although there is no evidence for the use of norepinephrine or serotonin as a retinal neurotransmitter in mice.

\section{Transcription factors defining transcriptionally related groups}

The ability to order AC types by transcriptomic similarity (Fig. $5 A$ ) led us to ask whether we could identify transcription factors expressed by groups of related types. We found a few transcription factors expressed by small groups of related types (e.g., Ebf3, Satb2 [Kay et al., 2011] and Etv1) as well as others expressed by single AC types [Sox2 (Whitney et al., 2014), Mafb and Nfib; Fig. $10 A]$. Many transcription factors expressed by ACs were also expressed by subsets of RGCs (Tran et al., 2019).

Of particular interest in this context were transcription factors selectively expressed by either GABAergic or glycinergic types.

$40 \mu \mathrm{m} . \mathbf{G}$, A single cluster (C17) expresses the cholinergic markers ChAT, Slc18a3 (VAChT), and $S / c 5 a 7$ (choline transporter). $\boldsymbol{H}$, Expression of genes encoding synthetic enzymes for monoamine neurotransmitters. $I$, Two AC clusters express Nos 1 and therefore could use NO as a transmitter.
We focused first on two such factors: Meis2 and Tcf4. Meis 2 was expressed at higher levels by most GABAergic types than by any glycinergic type (Fig. 10A), a pattern supported by immunohistochemical analysis using the canonical GABAergic and glycinergic markers. MEIS2 was present in $78.1 \pm 2.3 \%$ of $\mathrm{GAD}^{+} \mathrm{ACs}$ and $94.3 \pm 1.7 \%$ of MEIS2 $^{+}$ACs coexpressed GAD (mean \pm SEM from five or more images from two animals; 332 cells scored). In addition, a small fraction of $\mathrm{MEIS}^{+}$cells coexpressed GAD and GLYT1 $(2.0 \pm 0.6 \%)$, suggesting that they could be dual-neurotransmitter ACs, and $3.2 \pm 1.0 \%$ were double negative (Fig. 10B,C).

In contrast, Tcf4 was expressed at higher levels by all glycinergic and nGnG-1-3 types than by any GABAergic type (Fig. 10A). Immunostaining confirmed TCF4 expression in $83.0 \pm 4.2 \%$ of $\mathrm{GLYT}^{+}{ }^{+}$ACs. Of TCF ${ }^{+}$cells, $71.7 \pm 3.8 \%$ were $\mathrm{GLYT}^{+}$, and $25.0 \pm 3.4 \%$ were both $\mathrm{GLYT}^{-}$and $\mathrm{GAD}^{-}$(417 cells scored; Fig. $10 D, E$ ); these likely represented $n G n G$ ACs. In addition, a small fraction of $\mathrm{TCF}^{+}{ }^{+}$cells coexpressed GAD and GLYT1 $(3.4 \pm 1.1 \%)$. Double-staining with anti-MEIS2 and anti-TCF4 confirmed that they were present in mutually exclusive AC subsets (Fig. 10F,G).

Finally, we analyzed Eomes (Tbr2) and NeuroD2, which were expressed by restricted subsets of GABAergic and glycinergic AC types, respectively (Fig. 10A). Eomes, previously studied as a marker for intrinsically photosensitive RGCs (Mao et al., 2014; Sweeney et al., 2014), was expressed at higher levels by six GABAergic types than by any glycinergic types, and all EOMES ${ }^{+}$ ACs were $\mathrm{GAD}^{+}$(Fig. 10H). As expected, all EOMES ${ }^{+}$ACs were also MEIS2 ${ }^{+}$(Fig. 10J,K). NeuroD2 was expressed at higher levels by six glycinergic and two $\mathrm{nGnG}$ types than by any GABAergic types. The majority of NEUROD2 ACs expressed GLYT1, confirming a previous report demonstrating NEUROD2 expression in a subset of glycinergic ACs (Cherry et al., 2011; Fig. 10I). NEUROD2 ${ }^{+}$ACs were mutually exclusive from MEIS2 ${ }^{+}$ACs, suggesting the GLYT1 ${ }^{-}$and NEUROD2 ${ }^{+}$ACs are likely to be $\mathrm{nGnG}$ ACs (Fig. 10L,M).

Together, these patterns suggest possible roles for MEIS2, TCF4, EOMES, and NEUROD2 in establishing or maintaining the GABAergic and glycinergic cell types or correlated features of the ACs that express them.

\section{Discussion}

\section{Mouse AC types}

The heterogeneity of ACs has been recognized since the time of Cajal (1893) and documented by multiple methods (Diamond, 2017). Minimally biased cell filling methods identified 29 AC types in rabbit (MacNeil and Masland, 1998) and at least 25 AC types in mouse (Badea and Nathans, 2004; Majumdar et al., 2009; Pang et al., 2012), and reconstruction from serial electron microscopic sections revealed 45 AC types in mouse retina (Helmstaedter et al., 2013). These studies were likely underpowered, in that they surveyed a few hundred cells (by light microscopy) or reconstructed a limited area (electron microscopy). Our survey, based on 32,523 cells, increases the number to $63 \mathrm{AC}$ types (Table 1 ).

While the cellular features of several AC types have been described in previous studies, the morphology and physiology of the majority of molecular types remain uncharacterized. Surprisingly, of the 10 most abundant types, 6 have not, to our knowledge, been subjects of previous studies (C1, C2, C5, C7C9; Table 1). The markers we have identified may provide a starting point for labeling or gaining genetic access to these ACs. 


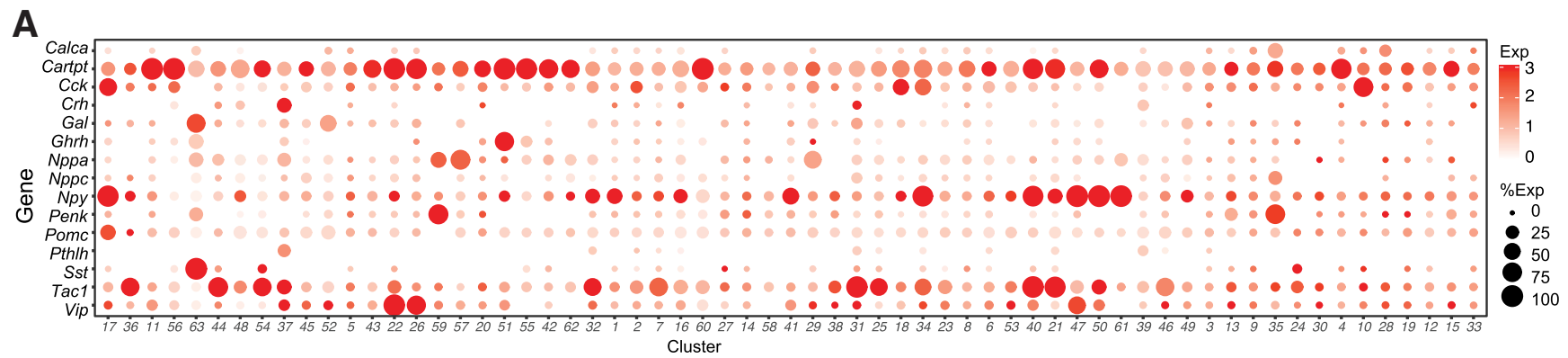

B

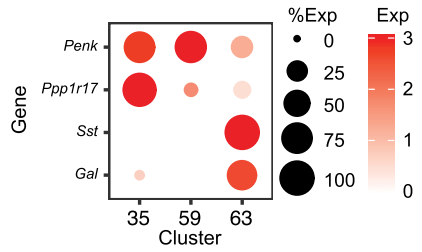

C CLUSTER 35 IHC

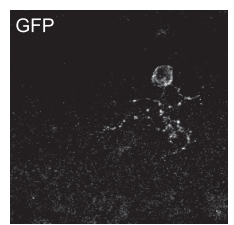

D CLUSTER 59

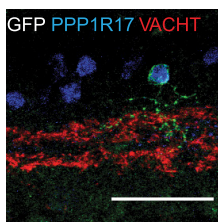

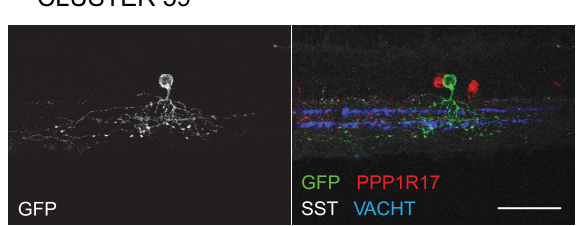

E

CLUSTER 63
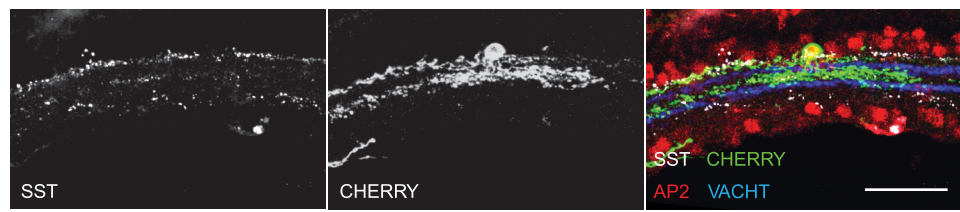

$\mathbf{F}$

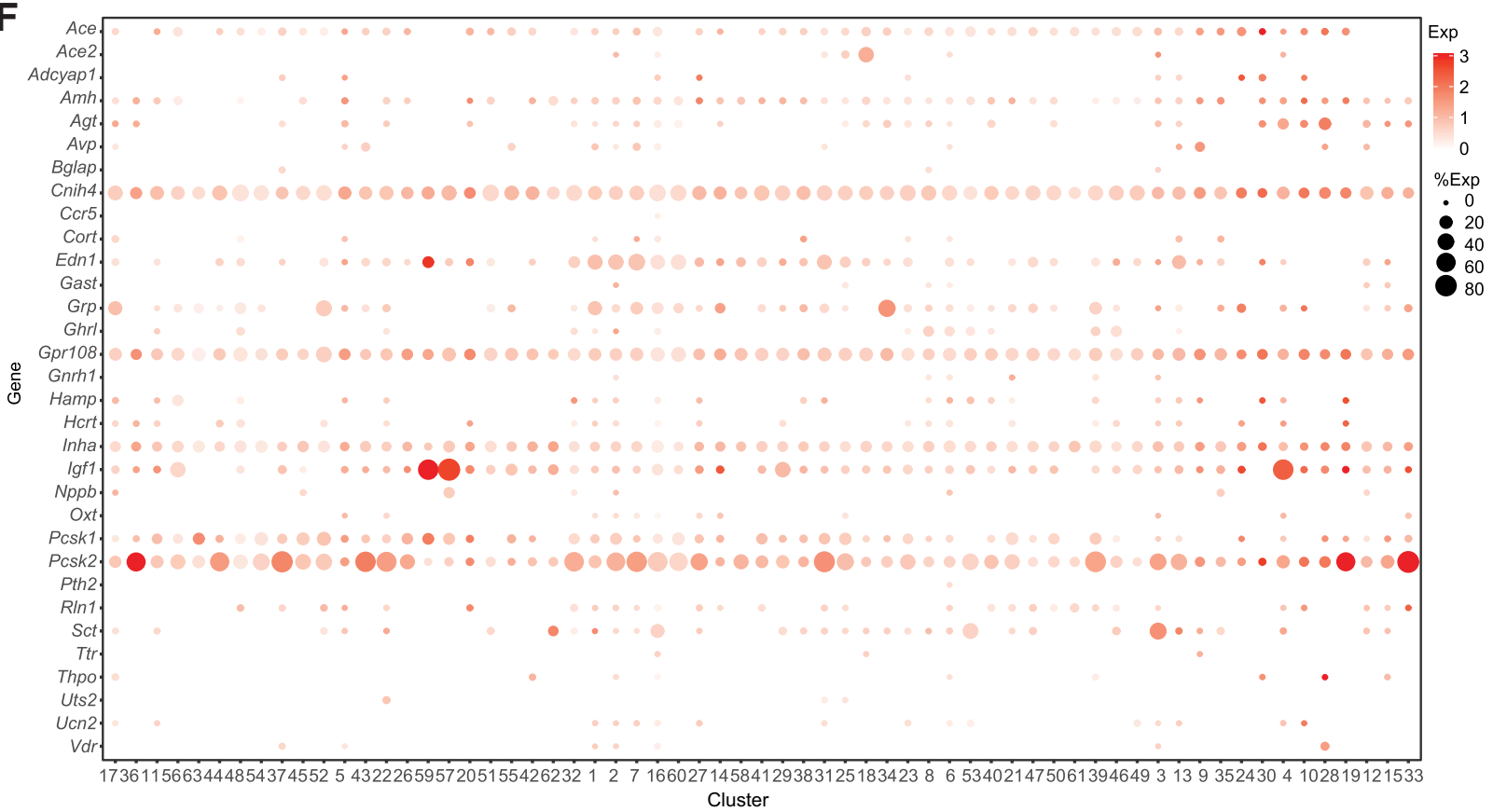

Figure 6. Many AC types express genes encoding neuromodulatory peptides. $A$, Dot plot showing peptides selectively expressed by one or a few $A C$ clusters. $B$, Markers that distinguish three $A C$ types that express the neuropeptide Penk. C-E, Penk ACs were visualized by sparse viral infection using a Cre-dependent Brainbow AAV reporter (AAV9-EF1a-BbTagBy or AAV9-EF1a-BbChrT) in Penk-cre mice and immunostaining with anti-GFP antibody. Laminae are highlighted by VAChT staining in $\boldsymbol{D}$ and $\boldsymbol{E}$; all amacrines are stained by AP2 in $E$. C, C35 ACs were identified by the coexpression of Penk and Ppp1r17. D, 559 ACs were Sst ${ }^{-}$and Ppp1r17 ${ }^{-}$. E, C63 ACs were Sst ${ }^{+}$. Scale bars: $\boldsymbol{C}, 20 \mu \mathrm{m} ; \boldsymbol{D}, \boldsymbol{E}, 40 \mu \mathrm{m}$. $\boldsymbol{F}$, Dot plot showing the expression of other neuropeptides in AC clusters.

Is the catalog now complete? We may have missed AC types for at least three reasons. First, types comprising $<0.1 \%$ of ACs might not have been detected, either because they were not collected or because our computational methods nominate clusters only when many cells share a transcriptional pattern. Second, we may have failed to collect some types for technical reasons (e.g., if they were particularly fragile). Third, some clusters could contain more than a single type. For example, our computational methods distinguish ON and OFF SACs as separate types in neonates (Peng et al., 2020), but their transcriptional 
A

\section{nGnG amacrine markers}

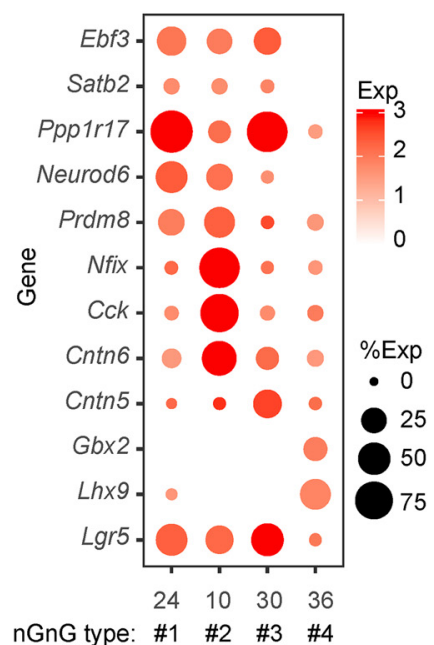

Cluster
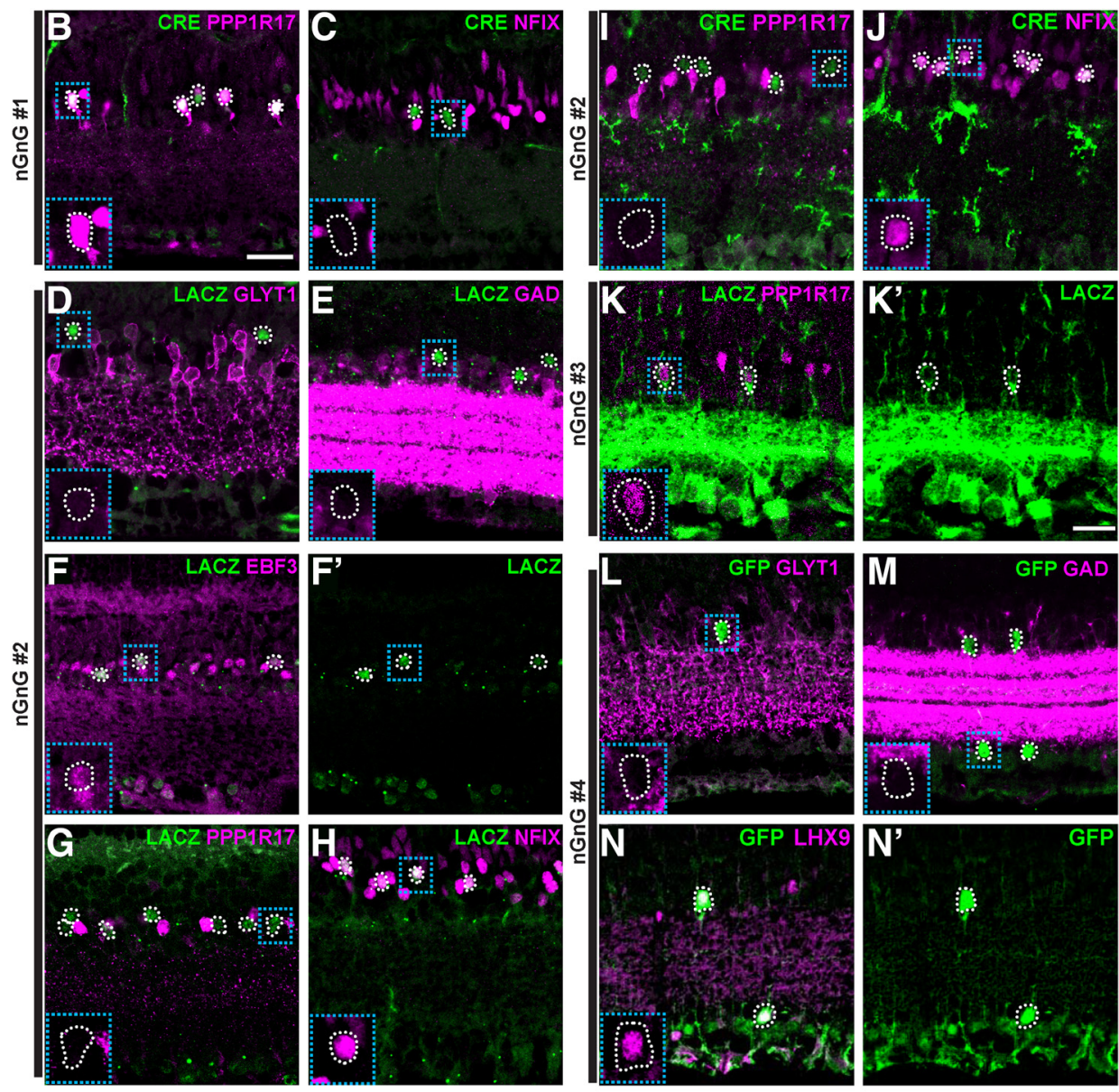

nGnG \#2
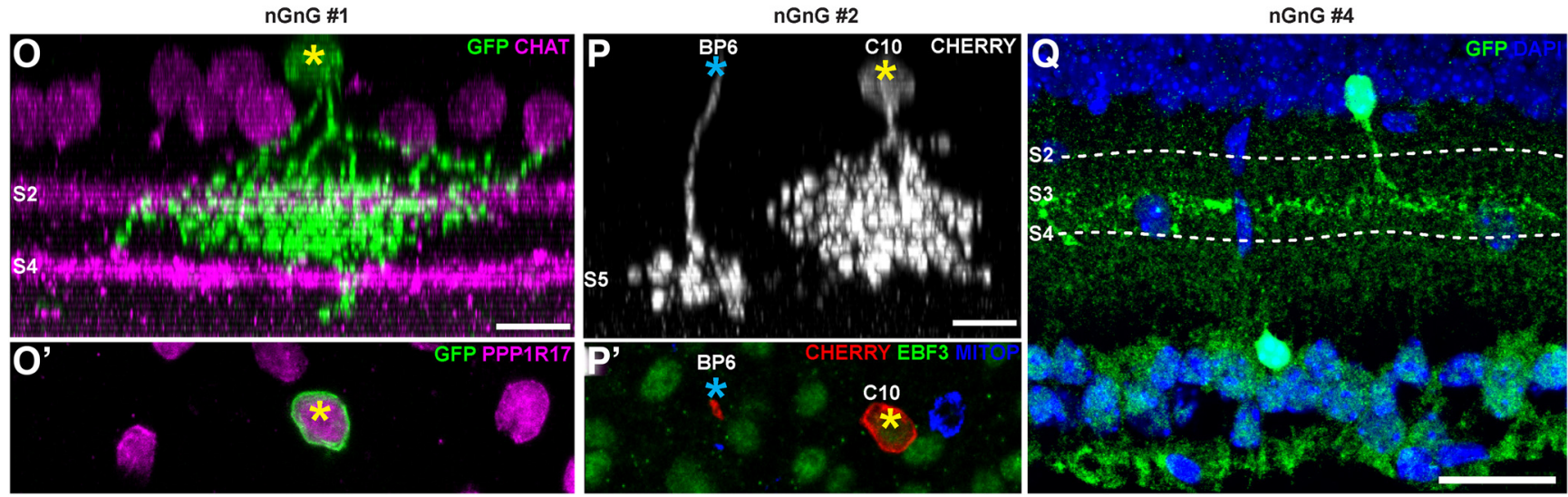

Figure 7. Molecular markers defining four types of $n G n G A C s . A$, Dot plot showing patterns of gene expression in four putative $n G n G$ AC types (nGnGs1-4: C24, C10, C30, and C36). $B, C$, nGnG-1 ACs (C24) labeled by immunostaining NeuroD6-cre retinas with an antibody against Cre recombinase together with either PPP1R17 or NFIX. nGnG1 ACs coexpressed PPP1R17 but were negative for NFIX. White dashed outlines indicate CRE $^{+}$cells. Blue dashed square inset shows $2 \times$ magnification. Scale bar, $25 \mu \mathrm{m}$. D-H, nGnG-2 ACs (C10) labeled in the Cntn6-LacZ line and detected by immunostaining with an antibody against LACZ were negative for GLYT1 $(\boldsymbol{D})$ and GAD65/67 (E), and coexpressed EBF3 (F) and NFIX (H), but were negative for PPP1R17 (G). $\boldsymbol{I}, J$, nGnG-2 ACs were also labeled using the Cck-IRES-cre and detected by staining for Cre recombinase. As when labeled by the Cntn6-LacZ line, most ACs labeled with this line were PPP1R17 and NFIX ${ }^{+} . K, n G n G-3$ ACs (C30) labeled in the Cntn5-Lacz line were detected by staining for LACZ and were PPP1R17 ${ }^{+}$. L-N, nGnG-4 ACs (C36) labeled in the Gbx2-CreER-GFP line and detected by immunostaining with an antibody against GFP, were GLYT1 ${ }^{-}(\boldsymbol{L})$ and GAD65/67- $(\boldsymbol{M})$, but LHX9 ${ }^{+}(\boldsymbol{N})$. $\mathbf{0}-\mathbf{Q}$, Dendritic lamination of nGnG ACs. $\mathbf{0}$, nGnG-1 morphology revealed as PPP1R17 cells in the Neurod6-Cre line. nGnG-1 ACs were visualized by sparse viral infection using a Cre-dependent Brainbow AAV reporter (AAV9-EF1a-BbTagBy). nGnG-1 ACs (yellow asterisk) laminated S1-3, as shown by ChAT staining (S2, S4). $0^{\prime}$ shows a $90^{\circ}$ rotated view of the soma of the labeled AC (yellow asterisk), confirming coexpression of PPP1R17. Scale bar, $10 \mu \mathrm{m}$. $\boldsymbol{P}$, nGnG-2 morphology revealed as EBF3 ${ }^{+}$cells, Mitop ${ }^{-}$(a mouse line that labels nGnG-1) ACs in the C(k-IRES-cre line. nGnG-2 ACs were visualized by sparse viral infection using a Cre-dependent Brainbow AAV reporter (AAV9-EF1a-BbTagBy). nGnG-2 ACs (yellow asterisk) laminated in S1-4, as demonstrated by its positioning relative to cone bipolar type 6 axon terminals (blue asterisk), which are also labeled in this line and laminate their axon terminals in $\mathrm{S5.} 0^{\prime}$ shows a $90^{\circ}$ rotated view of the labeled $\mathrm{AC}$ 's soma (yellow asterisk), confirming that the labeled $\mathrm{AC}$ was $\mathrm{EBF} 3^{+}$, Mitop $^{-}$(detected by GFP staining). Scale bar, $10 \mu \mathrm{m}$. Q, nGnG-4 lamination pattern revealed using the Gbx2-CreER-GFP line, GFP staining showed an AC that tightly laminated in S3. Sublaminae S2 and S4, as determined by Gad65/67 staining (data not shown), are represented by dashed white lines. Scale bar, $25 \mu \mathrm{m}$. 


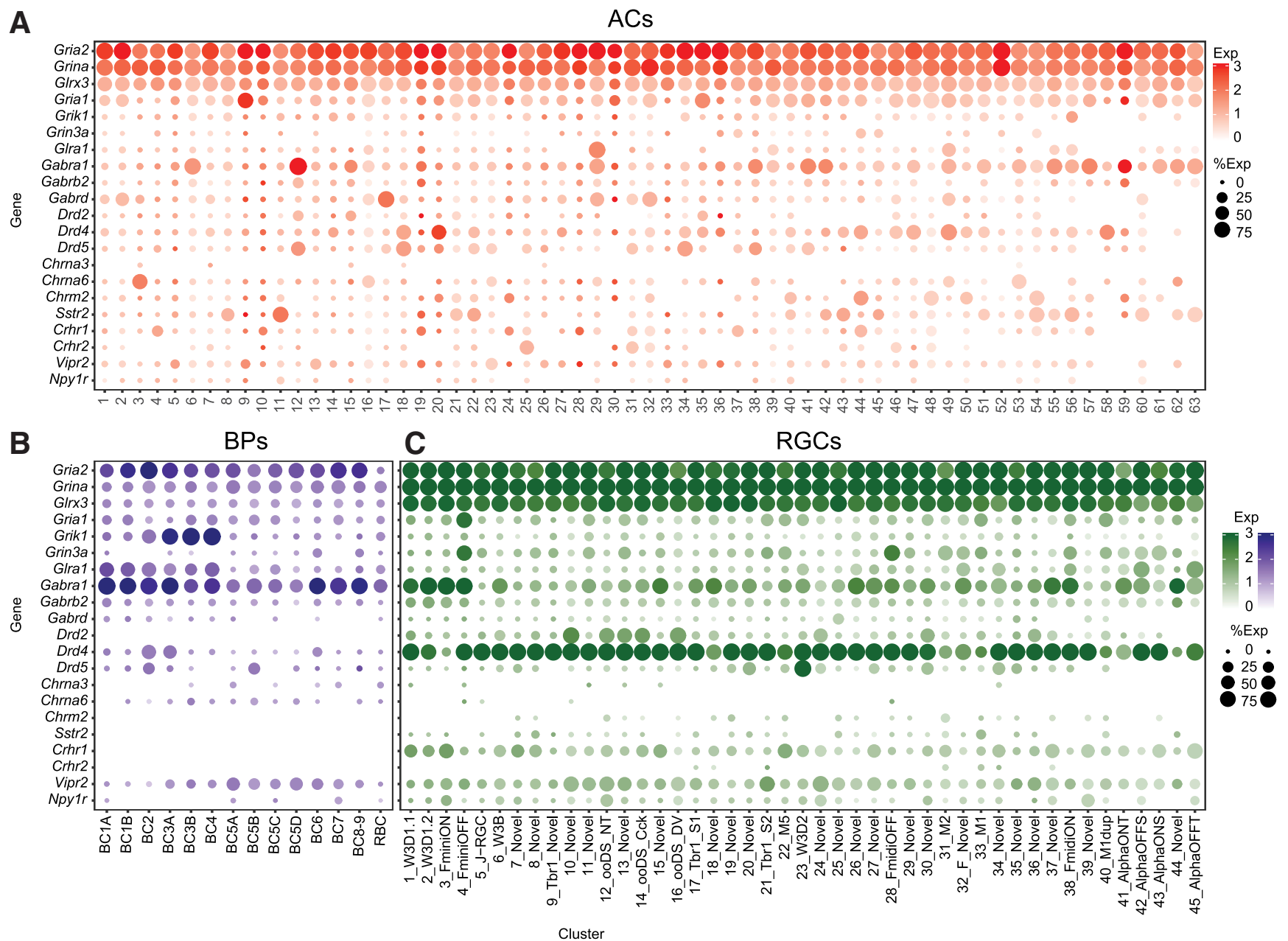

Figure 8. Expression of neurotransmitter and neuromodulator receptors in $\mathrm{ACS}, \mathrm{BC}$, and RGCS. $\boldsymbol{A}-\boldsymbol{C}$, Dot plots showing selected neurotransmitter and neuromodulator receptor expression in ACs $(\boldsymbol{A}$, red), BCs ( $\boldsymbol{B}$, dark blue; Shekhar et al., 2016), and RGCs ( $\boldsymbol{C}$, dark green; Tran et al., 2019). Some receptors were broadly expressed in most cell classes, some were selective to a certain class, and others were specific to certain types in certain classes. Expression of additional receptors in ACs is shown in Figure 9.

differences diminish with age, and they form a single cluster at P18-P19. Thus, we view 63 as a lower limit to the number of AC types but have no reason to expect that the true number greatly exceeds 63 .

\section{Neurotransmitters and neuromodulators}

ACs are largely inhibitory using GABA and glycine as neurotransmitters (Wässle et al., 2009b; Masland, 2012; Diamond, 2017). Our results support this dogma, with 56 of the 63 AC types being either GABAergic (43) or glycinergic (13) based on expressing Gad1/2 or Glyt1. In addition, however, we found three types that express both GABAergic and glycinergic markers at levels comparable to types expressing a single neurotransmitter. Although one of these types could be artifactual (composed of two ACs, doublets, that were profiled together; see Results), it is unlikely for the others (see Materials and Methods). How these types use each transmitter remains to be determined. We also found four types that express low levels of both Gad1/2 and Glyt1. These nonGABAergic nonglycinergic types, which we call nGnG1-4, include a previous type (Kay et al., 2011; Macosko et al., 2015) and three additional types. We confirmed a lack of detectable Gad and Glyt1 immunohistochemically, but physiological studies will be needed to determine whether low expression levels detected at the RNA level could nonetheless mediate GABAergic or glycinergic transmission. We found no evidence for the use of other small- molecule neurotransmitters by these ACs, but all AC types, including $\mathrm{nGnG}$ ACs, express neuropeptides.

Some AC types have been shown to use glutamate, acetylcholine, dopamine, or $\mathrm{NO}$ in tandem with GABA or glycine (Diamond, 2017). We identified AC types likely to use all of these transmitters, including a potentially novel glutamatergic type (VG1). The glutamatergic VG3 type is also glycinergic, as shown physiologically (Lee et al., 2016; Tien et al., 2016), whereas the VG1 type is likely GABAergic. We found no evidence that specific AC types synthesize other small-molecule neurotransmitters, including serotonin, tyramine, histamine, epinephrine, or norepinephrine. Some of these have been reported to be used by ACs in other species (Ghai et al., 2009), but in the majority of cases, conclusions are based on the uptake of or responsiveness to exogenous transmitter rather than the production of endogenous transmitter (Fletcher and Wässle, 1999).

In summary, ACs use a remarkable array of neurotransmitters and neuromodulators, and most AC types are likely capable of releasing at least two such bioactive species, thereby enhancing the range of signals they can provide (Nusbaum et al., 2017). Finally, although most neurotransmitter and neuropeptide receptors were broadly expressed in ACs, BCs, and RGCs, some were selectively expressed by a small number of neuronal types. Comparing their expression with that of neurotransmitter 


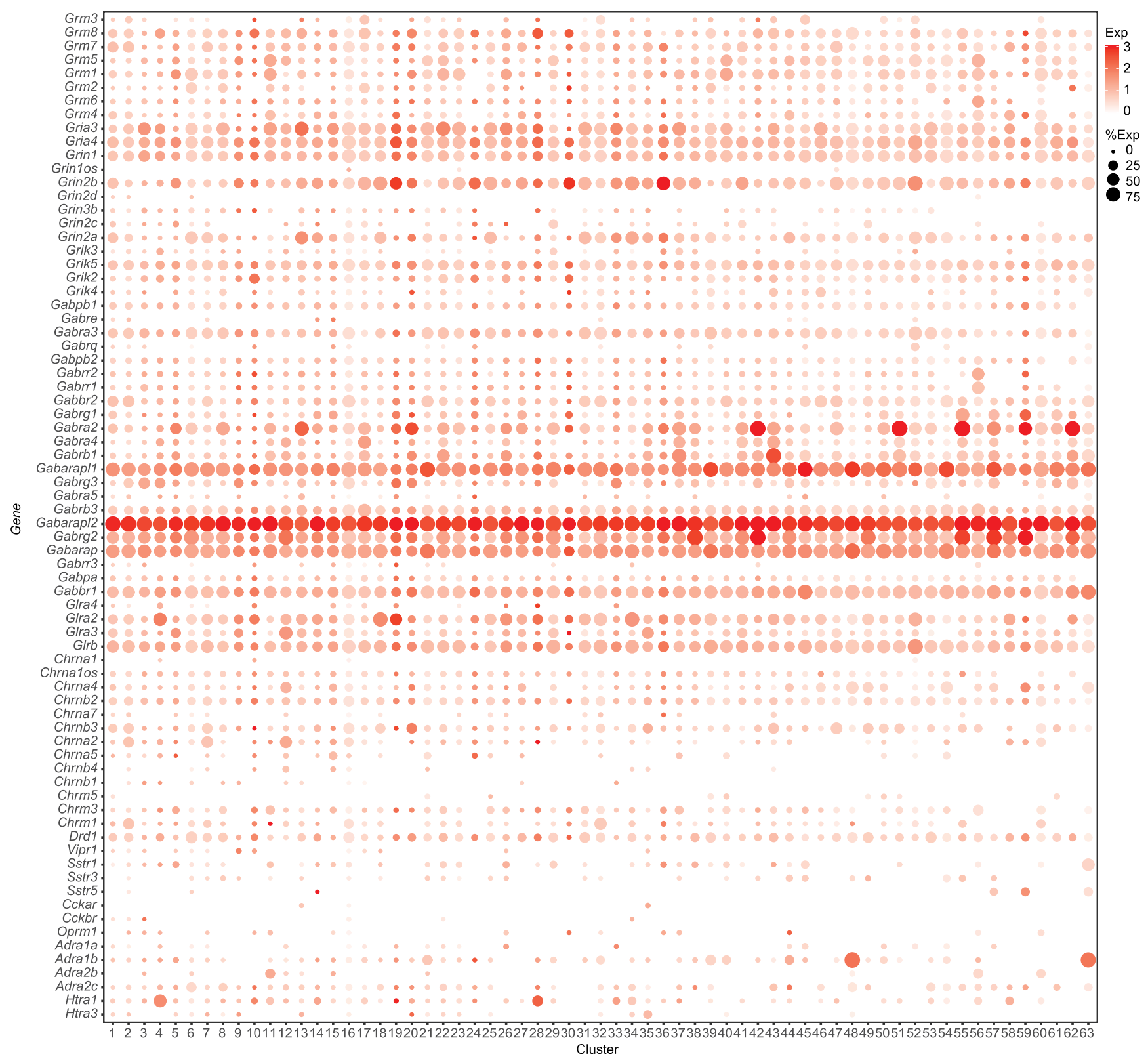

Figure 9. Expression of neurotransmitter and neuropeptide receptors in ACs. Dot plot showing the expression of neurotransmitter and neuropeptide receptors in ACs.

synthetic enzymes and neuropeptide genes in ACs can help guide attempts to elucidate synaptic connectivity.

\section{Transcriptional relationships among AC types}

Arranging AC types by transcriptional similarity (Figs. 5, 10) revealed several interesting relationships. First, the most fundamental (highest level) division is into GABAergic and glycinergic types. It is noteworthy that these two subclasses differ in additional ways: For example, most GABAergic ACs have relatively broad dendritic arbors confined to one or a few sublaminae in the IPL, whereas most glycinergic ACs have narrow arbors that span multiple sublaminae (Wässle et al., 2009b; Diamond, 2017). Thus, global comparison of genes differentially expressed by GABAergic and glycinergic types, such as the transcription factors discussed below, could reveal determinants of their contrasting morphologies as well as their transmitter choice.

Second, among GABAergic and glycinergic types, the most divergent are SACs and AII ACs, respectively. In fact, although the GABAergic nature of SACs is indisputable, they are no more closely related to other GABAergic than to glycinergic types (Figure 5A), and SACs are as closely related to RGCs as to other ACs (Macosko et al., 2015). SACs are among the first born during embryogenesis (Voinescu et al., 2009), and play an organizing role in patterning the sublamination of the IPL (Peng et al., 2017; Duan et al., 2018). SACs appear to be among the most evolutionarily ancient of AC types (Brandon, 1991; Criswell and Brandon, 1992; Peng et al., 2020), and AII ACs are unique in providing the principle route through which input from rods is delivered to retinal ganglion cells (Demb and Singer, 2012).

Third, some AC types with similar transmitter profiles are close transcriptional relatives, but this is not always the case. For example, three of the $\mathrm{nGnG}$ types are close relatives of each other, but one type is distant. nGnG-1 is also closely related to the glycinergic SEG AC, consistent with our previous demonstration that a postmitotic fate choice diversifies these two (Kay et al., 2011). Some peptide-expressing clusters are closely related, 
A

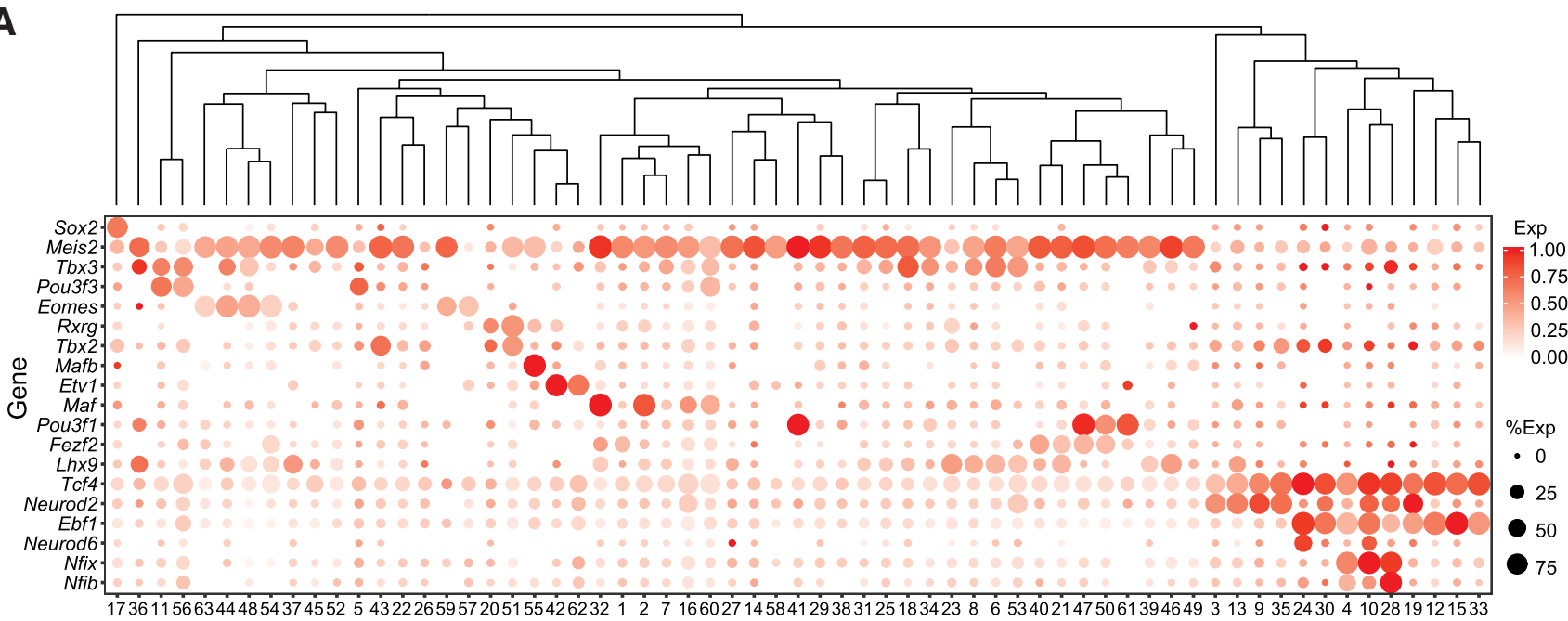

Cluster
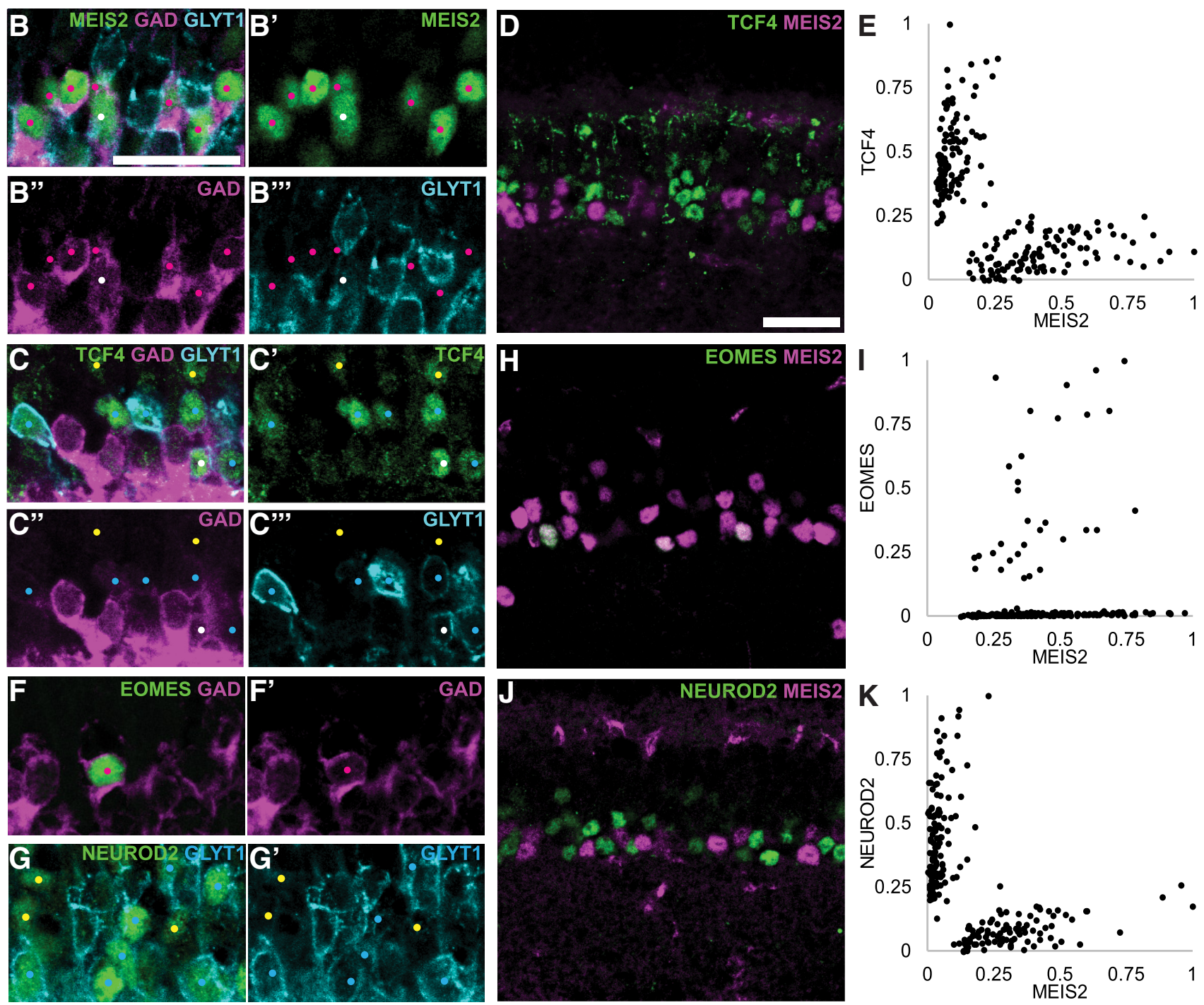

Figure 10. Transcription factors expressed by ACs. A, Dot plot showing row-normalized expression of selected transcription factors by AC types. Dendrogram shows transcriptional relationships among AC clusters as determined by hierarchical clustering of average gene signatures (Euclidean distance metric, average linkage). Color indicates the average normalized transcript level per cluster in expressing cells. $\boldsymbol{B}$, Immunostaining shows that MEIS2 ${ }^{+}$ACs (dots) were largely GAD ${ }^{+}$(detected by an anti-GAD65/67 antibody) and GlyT1 ${ }^{-}$(magenta dots) in P21 mouse retina inner nuclear layer (INL), a rare population of MEIS2 ${ }^{+} \mathrm{ACS}$, were double positive for these markers (white dots). Scale bar, $25 \mu \mathrm{m}$. C, The majority of TCF4 ${ }^{+} \mathrm{ACS}$ were $\mathrm{GLYT1}^{+}$, GAD ${ }^{-}$(magenta dots). A subset was double negative (yellow dots) and a rare population was double positive (white dots). $\boldsymbol{D}$, MEIS2 and TCF4 mark mutually exclusive groups of ACs. Scale bar, $25 \mu \mathrm{m}$. $\boldsymbol{E}$, 
Table 2. The mouse retinal cell atlas

\begin{tabular}{lcc}
\hline Class & Types in $2010(n)$ & Types in $2020(n)$ \\
\hline PR & 3 & 3 \\
HC & 1 & 1 \\
BC & 12 & 15 \\
RGC & $\sim 20$ & 46 \\
AC & $\sim 30$ & 63 \\
Total & $\sim 65$ & 128
\end{tabular}

Number of types per class estimated in 2010 (Wässle et al., 2009a; Masland, 2012) and current estimate based on data in this study, Macosko et al. (2015), Shekhar et al. (2016), and Tran et al. (2019).

for example, two of the $\mathrm{VIP}^{+}$types and two of the $\mathrm{CCK}^{+}$types. In contrast, the shared expression of other neurotransmitters or neuromodulators is not reflected in overall transcriptional similarity. Thus, two other CCK-expressing types are distant relatives of each other, and the two putative glutamatergic types (VG1 and VG3) are not close relatives.

Fourth, several transcription factors, including Meis2, Tcf4, Ebf1, Neurod2, and Eomes (Tbr2) are expressed by groups of closely related GABAergic (Meis2, Eomes) or glycinergic (Tcf4, Ebf1, Neurod2) AC types. Association of some of these genes with GABAergic (Meis2: Bumsted-O'Brien et al., 2007) or glycinergic (NeuroD2: Cherry et al., 2011) ACs has been noted previously, but comprehensive analysis of their expression has not been reported. Of these, Meis 2 and Tcf4 are of particular interest, since they are expressed by most GABAergic and all glycinergic ACs, respectively. Both transcription factors play critical developmental roles in multiple cell types, both within and outside of the nervous system (Tcf4: Forrest et al., 2014; Meis2: Schulte and Geerts, 2019), but their roles in retinal development have not been reported to date.

The patterns we documented raise the tantalizing possibility that selectively expressed transcription factors play roles in specifying AC types or subclasses, or in the determining features they share. It will be possible to test these possibilities using genetic methods that have been applied successfully to transcriptional regulators in other retinal cell types.

\section{The mouse retinal cell atlas}

We previously analyzed all retinal cell classes other than amacrines, documenting the existence of 65 neuronal types and 6 non-neuronal types (astrocytes, endothelial cells, fibroblasts, microglia, Müller glia, and pericytes; Macosko et al., 2015; Shekhar et al., 2016; Tran et al., 2019). To these, we now add 63 AC types. Thus, the mouse retinal atlas stands at 128 neuronal types (Table 2) and a total of at least 134 cell types.

The existence of six major classes of retinal cells (five neuronal plus Müller glia) was clear nearly 130 years ago (Cajal, 1893). Over the subsequent years, classical studies in numerous laboratories and of numerous species defined many types within classes, but the low-throughput nature of the available methods

$\leftarrow$

Quantification of fluorescent intensity of MEIS2 ( $x$-axis) and TCF4 ${ }^{+}$(y-axis) cells in the INL at P21. Raw fluorescent intensity values were background subtracted and normalized to the maximum intensity value for each marker (225 cells scored). $\boldsymbol{F}$, EOMES ${ }^{+}$cells in the INL were $\mathrm{GAD}^{+}$(magenta dot). $\mathbf{G}$, The majority of NEUROD2 ${ }^{+}$cells in the INL are GLYT1 ${ }^{+}$(blue dot), and a subset is $\mathrm{GLYT1}^{-}$(yellow dots). $\boldsymbol{H}, \mathrm{EOMES}^{+} \mathrm{ACs}$ are a subset of MEIS2 ${ }^{+} \mathrm{ACs}$. $\boldsymbol{I}_{\text {, }}$ Quantification of fluorescence intensity of MEIS2 ${ }^{+}$( $x$-axis) and EOMES ${ }^{+}$( $y$-axis) cells in the INL at P21 (217 cells scored). J, NEUROD2 ${ }^{+}$ACs are MEIS2 ${ }^{-}$. $\boldsymbol{K}$, Quantification of fluorescence intensity of $\mathrm{MEIS2}^{+}$(x-axis) and NEUROD2 ${ }^{+}$(y-axis) cells in the INL at P21 (222 cells scored). prevented the generation of a comprehensive inventory. In an influential and authoritative review published less than a decade ago, the number of retinal cell types was estimated to be $<70$ (Masland, 2012; Table 2). Since that time, the estimated number has nearly doubled, largely as a result of applying newly developed high-throughput ultrastructural, physiological, and, above all, transcriptomic methods. Although it is too soon to declare victory, we believe that the current number is close to accurate.

\section{References}

Akrouh A, Kerschensteiner D (2015) Morphology and function of three VIPexpressing amacrine cell types in the mouse retina. J Neurophysiol 114:2431-2438.

Badea TC, Nathans J (2004) Quantitative analysis of neuronal morphologies in the mouse retina visualized by using a genetically directed reporter. J Comp Neurol 480:331-351.

Baden T, Berens P, Franke K, Rosón MR, Bethge M, Euler T (2016) The functional diversity of retinal ganglion cells in the mouse. Nature 529:345350 .

Bae JA, Mu S, Kim JS, Turner NL, Tartavull I, Kemnitz N, Jordan CS, Norton AD, Silversmith WM, Prentki R, Sorek M, David C, Jones DL, Bland D, Sterling ALR, Park J, Briggman KL, Seung HS (2018) Digital museum of retinal ganglion cells with dense anatomy and physiology. Cell 173:12931306.e19.

Boehning D, Snyder SH (2003) Novel neural modulators. Annu Rev Neurosci 26:105-131.

Brandon C (1991) Cholinergic amacrine neurons of the dogfish retina. Vis Neurosci 6:553-562.

Buffelli M, Burgess RW, Feng G, Lobe CG, Lichtman JW, Sanes JR (2003) Genetic evidence that relative synaptic efficacy biases the outcome of synaptic competition. Nature 424:430-434.

Bumsted-O'Brien KM, Hendrickson A, Haverkamp S, Ashery-Padan R, Schulte D (2007) Expression of the homeodomain transcription factor Meis2 in the embryonic and postnatal retina. J Comp Neurol 505:58-72.

Cai D, Cohen KB, Luo T, Lichtman JW, Sanes JR (2013) Improved tools for the Brainbow toolbox. Nat Methods 10:540-547.

Cajal SR (1893) y La rétine des vertébrés. La cellule 9:119-257.

Chen J, Lin Y, Huang J, Wang W, Wei Y-Y, Li Y-Q, Kaneko T, Wu S-X (2013) Mammal retinal distribution of ENKergic amacrine cells and their neurochemical features: evidence from the PPE-GFP transgenic mice. Neurosci Lett 548:233-238.

Chen L, Guo Q, Li JY (2009) Transcription factor Gbx2 acts cell-nonautonomously to regulate the formation of lineage-restriction boundaries of the thalamus. Development 136:1317-1326.

Chen M, Tian S, Glasgow NG, Gibson G, Yang X, Shiber CE, Funderburgh J, Watkins S, Johnson JW, Schuman JS, Liu H (2015) Lgr5+ amacrine cells possess regenerative potential in the retina of adult mice. Aging Cell 14:635-643.

Chen T, Guestrin C (2016) Xgboost: a scalable tree boosting system. In: Proceedings of the 22nd ACM SIGKDD International Conference on Knowledge Discovery and Data Mining, pp 785-794. New York: Association for Computing Machinery.

Cherry TJ, Wang S, Bormuth I, Schwab M, Olson J, Cepko CL (2011) NeuroD factors regulate cell fate and neurite stratification in the developing retina. J Neurosci 31:7365-7379.

Criswell MH, Brandon C (1992) Cholinergic and GABAergic neurons occur in both the distal and proximal turtle retina. Brain Res 577:101-111.

Demb JB, Singer JH (2012) Intrinsic properties and functional circuitry of the AII amacrine cell. Vis Neurosci 29:51-60.

Diamond JS (2017) Inhibitory interneurons in the retina: types, circuitry, and function. Annu Rev Vis Sci 3:1-24.

Duan X, Krishnaswamy A, Laboulaye MA, Liu J, Peng YR, Yamagata M, Toma K, Sanes JR (2018) Cadherin combinations recruit dendrites of distinct retinal neurons to a shared interneuronal scaffold. Neuron 99:11451154.

Finak G, McDavid A, Yajima M, Deng J, Gersuk V, Shalek AK, Slichter CK, Miller HW, McElrath MJ, Prlic M, Linsley PS, Gottardo R (2015) MAST: a flexible statistical framework for assessing transcriptional changes and characterizing heterogeneity in single-cell RNA sequencing data. Genome Biol 16:278. 
Firth SI, Varela C, De La Villa P, Marshak DW (2002) Cholecystokinin-like immunoreactive amacrine cells in the rat retina. Vis Neurosci 19:531540 .

Fletcher EL, Wässle H (1999) Indoleamine-accumulating amacrine cells are presynaptic to rod bipolar cells through $\mathrm{GABA}(\mathrm{C})$ receptors. J Comp Neurol 413:155-167.

Forrest MP, Hill MJ, Quantock AJ, Martin-Rendon E, Blake DJ (2014) The emerging roles of TCF4 in disease and development. Trends Mol Med 20:322-331.

Franke K, Berens P, Schubert T, Bethge M, Euler T, Baden T (2017) Inhibition decorrelates visual feature representations in the inner retina. Nature 542:439-444.

Fujitani Y, Fujitani S, Luo H, Qiu F, Burlison J, Long Q, Kawaguchi Y, Edlund H, MacDonald RJ, Furukawa T, Fujikado T, Magnuson MA, Xiang M, Wright CVE (2006) Ptfla determines horizontal and amacrine cell fates during mouse retinal development. Development 133:44394450 .

Ghai K, Zelinka C, Fischer AJ (2009) Serotonin released from amacrine neurons is scavenged and degraded in bipolar neurons in the retina. J Neurochem 111:1-14.

Goebbels S, Bormuth I, Bode U, Hermanson O, Schwab MH, Nave KA (2006) Genetic targeting of principal neurons in neocortex and hippocampus of NEX-Cre mice. Genesis 44:611-621.

Greene MJ, Kim JS, Seung HS (2016) Analogous convergence of sustained and transient inputs in parallel on and off pathways for retinal motion computation. Cell Rep 14:1892-1900.

Grimes WN, Zhang J, Graydon CW, Kachar B, Diamond JS (2010) Retinal parallel processors: more than 100 independent microcircuits operate within a single interneuron. Neuron 65:873-885.

Grimes WN, Seal RP, Oesch N, Edwards RH, Diamond JS (2011) Genetic targeting and physiological features of VGLUT3+ amacrine cells. Vis Neurosci 28:381-392.

Hansen KA, Torborg CL, Elstrott J, Feller MB (2005) Expression and function of the neuronal gap junction protein connexin 36 in developing mammalian retina. J Comp Neurol 493:309-320.

Harris JA, Hirokawa KE, Sorensen SA, Gu H, Mills M, Ng LL, Bohn P, Mortrud M, Ouellette B, Kidney J, Smith KA, Dang C, Sunkin S, Bernard A, Oh SW, Madisen L, Zeng H (2014) Anatomical characterization of Cre driver mice for neural circuit mapping and manipulation. Front Neural Circuits 8:76.

Haverkamp S, Wässle H (2004) Characterization of an amacrine cell type of the mammalian retina immunoreactive for vesicular glutamate transporter 3. J Comp Neurol 468:251-263.

Helmstaedter M, Briggman KL, Turaga SC, Jain V, Seung HS, Denk W (2013) Connectomic reconstruction of the inner plexiform layer in the mouse retina. Nature 500:168-174.

Jacoby J, Zhu Y, DeVries SH, Schwartz GW (2015) An amacrine cell circuit for signaling steady illumination in the retina. Cell Rep 13:2663-2670.

Jacoby J, Nath A, Jessen ZF, Schwartz GW (2018) A self-regulating gap junction network of amacrine cells controls nitric oxide release in the retina. Neuron 100:1149-1162.e5.

Jeon C-J, Strettoi E, Masland RH (1998) The major cell populations of the mouse retina. J Neurosci 18:8936-8946.

Johnson J, Sherry DM, Liu X, Fremeau RT Jr, Seal RP, Edwards RH, Copenhagen DR (2004) Vesicular glutamate transporter 3 expression identifies glutamatergic amacrine cells in the rodent retina. J Comp Neurol 477:386-398.

Karten HJ, Brecha N (1983) Localization of neuroactive substances in the vertebrate retina: evidence for lamination in the inner plexiform layer. Vision Res 23:1197-1205.

Kawaguchi Y, Cooper B, Gannon M, Ray M, MacDonald RJ, Wright CV (2002) The role of the transcriptional regulator Ptfla in converting intestinal to pancreatic progenitors. Nat Genet 32:128-134.

Kay JN, Voinescu PE, Chu MW, Sanes JR (2011) Neurod6 expression defines new retinal amacrine cell subtypes and regulates their fate. Nat Neurosci 14:965-972.

Keeley PW, Reese BE (2018) DNER and NFIA are expressed by developing and mature AII amacrine cells in the mouse retina. J Comp Neurol 526:467-479.

Kerstein PC, Leffler J, Sivyer B, Taylor WR, Wright KM (2020) Gbx2 identifies two amacrine cell subtypes with distinct molecular, morphological, and physiological properties. bioRxiv. Available at https://doi.org/ 10.1101/2020.05.19.104307.

Krishnaswamy A, Yamagata M, Duan X, Hong YK, Sanes JR (2015) Sidekick 2 directs formation of a retinal circuit that detects differential motion. Nature 524:466-470.

Laboulaye MA, Duan X, Qiao M, Whitney IE, Sanes JR (2018) Mapping transgene insertion sites reveals complex interactions between mouse transgenes and neighboring endogenous genes. Front Mol Neurosci 11:385.

Lakowski J, YT, Han RA, Pearson A, Gonzalez -Cordero E, West S, Gualdoni A, Barber M, Hubank R, Ali J Sowden (2011) Effective transplantation of photoreceptor precursor cells selected via cell surface antigen expression. Stem Cells 29:1391-1404.

Lee S, Zhang Y, Chen M, Zhou ZJ (2016) Segregated glycine-glutamate cotransmission from vGluT3 amacrine cells to contrast-suppressed and contrast-enhanced retinal circuits. Neuron 90:27-34.

Leung C, Tan SH, Barker N (2018) Recent advances in Lgr5+ stem cell research. Trends Cell Biol 28:380-391.

Li H, Takeda Y, Niki H, Ogawa J, Kobayashi S, Kai N, Akasaka K, Asano M, Sudo K, Iwakura Y, Watanabe K (2003) Aberrant responses to acoustic stimuli in mice deficient for neural recognition molecule NB-2. Eur J Neurosci 17:929-936

Lin B, Masland RH (2006) Populations of wide-field amacrine cells in the mouse retina. J Comp Neurol 499:797-809.

MacNeil MA, Masland RH (1998) Extreme diversity among amacrine cells: implications for function. Neuron 20:971-982.

Macosko EZ, Basu A, Satija R, Nemesh J, Shekhar K, Goldman M, Tirosh I, Bialas AR, Kamitaki N, Martersteck EM, Trombetta JJ, Weitz DA, Sanes JR, Shalek AK, Regev A, McCarroll SA (2015) Highly parallel genomewide expression profiling of individual cells using nanoliter droplets. Cell 161:1202-1214.

Majumdar S, Weiss J, Wässle H (2009) Glycinergic input of widefield, displaced amacrine cells of the mouse retina. J Physiol 587:3831-3849.

Mao C-A, Li H, Zhang Z, Kiyama T, Panda S, Hattar S, Ribelayga CP, Mills SL, Wang SW (2014) T-box transcription regulator Tbr2 is essential for the formation and maintenance of Opn4/melanopsin-expressing intrinsically photosensitive retinal ganglion cells. J Neurosci 34:13083-13095.

Martersteck EM, Hirokawa KE, Evarts M, Bernard A, Duan X, Li Y, Ng L, Oh SW, Ouellette B, Royall JJ, Stoecklin M, Wang Q, Zeng H, Sanes JR, Harris JA (2017) Diverse central projection patterns of retinal ganglion cells. Cell Rep 18:2058-2072.

Masland RH (2012) The neuronal organization of the retina. Neuron 76:266280.

McGinnis CS, Murrow LM, Gartner ZJ (2019) DoubletFinder: doublet detection in single-cell RNA sequencing data using artificial nearest neighbors. Cell Syst 8:329-337.

Misgeld T, Kerschensteiner M, Bareyre FM, Burgess RW, Lichtman JW (2007) Imaging axonal transport of mitochondria in vivo. Nat Methods 4:559-561.

Nusbaum MP, Blitz DM, Marder E (2017) Functional consequences of neuropeptide and small-molecule co-transmission. Nat Rev Neurosci 18:389-403.

Pandey S, Shekhar K, Regev A, Schier AF (2018) Comprehensive Identification and Spatial Mapping of Habenular Neuronal Types Using Single-Cell RNA-Seq. Curr Biol 28:1052-1065.e7.

Pang JJ, Gao F, Wu SM (2010) Light responses and morphology of bNOSimmunoreactive neurons in the mouse retina. J Comp Neurol 518:24562474.

Pang JJ, Gao F, Wu SM (2012) Physiological characterization and functional heterogeneity of narrow-field mammalian amacrine cells. J Physiol 590:223-234.

Park SJ, Borghuis BG, Rahmani P, Zeng Q, Kim I-J, Demb JB (2015) Function and circuitry of $\mathrm{VIP}^{+}$interneurons in the mouse retina. J Neurosci 35:10685-10700.

Park SJ, Pottackal J, Ke J-B, Jun NY, Rahmani P, Kim I-J, Singer JH, Demb JB (2018) Convergence and divergence of CRH amacrine cells in mouse retinal circuitry. J Neurosci 38:3753-3766.

Peng Y-R, Tran NM, Krishnaswamy A, Kostadinov D, Martersteck EM, Sanes JR (2017) Satb1 regulates contactin 5 to pattern dendrites of a mammalian retinal ganglion cell. Neuron 95:869-883.

Peng Y-R, Shekhar K, Yan W, Herrmann D, Sappington A, Bryman GS, Van Zyl T, Do MTH, Regev A, Sanes JR (2019) Molecular classification and 
comparative taxonomics of foveal and peripheral cells in primate retina. Cell 176:1222-1237.e22.

Peng Y-R, James RE, Yan W, Kay JN, Kolodkin AL, Sanes JR (2020) Binary fate choice between closely related interneuronal types is determined by a fezf1-dependent postmitotic transcriptional switch. Neuron 105:464-474. e6.

Pérez de Sevilla Müller L, Azar SS, de los Santos J, Brecha NC (2017) Prox1 is a marker for AII amacrine cells in the mouse retina. Front Neuroanat 11:39.

Pérez de Sevilla Müller L, Solomon A, Sheets K, Hapukino H, Rodriguez AR, Brecha NC (2019) Multiple cell types form the VIP amacrine cell population. J Comp Neurol 527:133-158.

Patterson N, Price AL, Reich D (2006) Population structure and eigenanalysis. PLoS Genet 2:e190.

Puthussery T, Fletcher E (2007) Neuronal expression of P2X3 purinoceptors in the rat retina. Neuroscience 146:403-414.

Rheaume BA, Jereen A, Bolisetty M, Sajid MS, Yang Y, Renna K, Sun L, Robson P, Trakhtenberg EF (2018) Single cell transcriptome profiling of retinal ganglion cells identifies cellular subtypes. Nat Commun 9:3203.

Rice DS, Curran T (2000) Disabled-1 is expressed in type AII amacrine cells in the mouse retina. J Comp Neurol 424:327-338.

Rowan S, Cepko CL (2004) Genetic analysis of the homeodomain transcription factor Chx10 in the retina using a novel multifunctional BAC transgenic mouse reporter. Dev Biol 271:388-402.

Sanes JR, Masland RH (2015) The types of retinal ganglion cells: current status and implications for neuronal classification. Annu Rev Neurosci 38:221-246.

Seabrook TA, Burbridge TJ, Crair MC, Huberman AD (2017) Architecture, function, and assembly of the mouse visual system. Annu Rev Neurosci 40:499-538.

Schulte D, Geerts D (2019) MEIS transcription factors in development and disease. Development 146:dev174706.

Shekhar K, Lapan SW, Whitney IE, Tran NM, Macosko EZ, Kowalczyk M, Adiconis X, Levin JZ, Nemesh J, Goldman M, McCarroll SA, Cepko CL, Regev A, Sanes JR (2016) Comprehensive classification of retinal bipolar neurons by single-cell transcriptomics. Cell 166:1308-1323.e30.

Sukhdeo K, Koch CE, Miller TE, Zhou H, Rivera M, Yan K, Cepko CL, Lathia JD, Rich JN (2014) The Lgr5 transgene is expressed specifically in glycinergic amacrine cells in the mouse retina. Exp Eye Res 119:106-110.

Sweeney NT, Tierney H, Feldheim DA (2014) Tbr2 is required to generate a neural circuit mediating the pupillary light reflex. J Neurosci 34:54475453.

Takeda Y, Akasaka K, Lee S, Kobayashi S, Kawano H, Murayama S, Takahashi N, Hashimoto K, Kano M, Asano M, Sudo K, Iwakura Y, Watanabe K (2003) Impaired motor coordination in mice lacking neural recognition molecule NB-3 of the contactin/F3 subgroup. J Neurobiol $56: 252-265$

Taniguchi H, He M, Wu P, Kim S, Paik R, Sugino K, Kvitsiani D, Kvitsani D, Fu Y, Lu J, Lin Y, Miyoshi G, Shima Y, Fishell G, Nelson SB, Huang ZJ (2011) A resource of Cre driver lines for genetic targeting of GABAergic neurons in cerebral cortex. Neuron 71:995-1013.

Theofilas P, Steinhäuser C, Theis M, Derouiche A (2017) Morphological study of a connexin 43-GFP reporter mouse highlights glial hetero- geneity, amacrine cells, and olfactory ensheathing cells. J Neurosci Res 95:2182-2194.

Tien N-W, Kim T, Kerschensteiner D (2016) Target-specific glycinergic transmission from VGluT3-expressing amacrine cells shapes suppressive contrast responses in the retina. Cell Rep 15:1369-1375.

Tran NM, Shekhar K, Whitney IE, Jacobi A, Benhar I, Hong G, Yan W, Adiconis X, Arnold ME, Lee JM, Levin JZ, Lin D, Wang C, Lieber CM, Regev A, He Z, Sanes JR (2019) Single-cell profiles of retinal ganglion cells differing in resilience to injury reveal neuroprotective genes. Neuron 104:1039-1055.

Vaney DI, Sivyer B, Taylor WR (2012) Direction selectivity in the retina: symmetry and asymmetry in structure and function. Nat Rev Neurosci 13:194-208.

van Zyl T, Yan W, McAdams A, Peng Y-R, Shekhar K, Regev A, Juric D, Sanes JR (2020) Cell atlas of aqueous humor outflow pathways in eyes of humans and four model species provides insight into glaucoma pathogenesis. Proc Natl Acad Sci U S A 117:10339-10349.

Voinescu PE, Emanuela P, Kay JN, Sanes JR (2009) Birthdays of retinal amacrine cell subtypes are systematically related to their molecular identity and soma position. J Comp Neurol 517:737-750.

Vuong HE, de Sevilla Müller LP, Hardi CN, McMahon DG, Brecha NC (2015) Heterogeneous transgene expression in the retinas of the TH-RFP, TH-Cre, TH-BAC-Cre and DAT-Cre mouse lines. Neuroscience 307:319-337.

Wässle H, Puller C, Müller F, Haverkamp S (2009a) Cone contacts, mosaics, and territories of bipolar cells in the mouse retina. J Neurosci 29:106117.

Wässle H, Heinze L, Ivanova E, Majumdar S, Weiss J, Harvey RJ, Haverkamp S (2009b) Glycinergic transmission in the Mammalian retina. Front Mol Neurosci 2:6.

Wei W (2018) Neural mechanisms of motion processing in the mammalian retina. Annu Rev Vis Sci 4:165-192.

Werblin FS (2010) Six different roles for crossover inhibition in the retina: correcting the nonlinearities of synaptic transmission. Vis Neurosci 27:1-

Whitney IE, Keeley PW, John AJS, Kautzman AG, Kay JN, Reese BE (2014) Sox2 regulates cholinergic amacrine cell positioning and dendritic stratification in the retina. J Neurosci 34:10109-10121.

Yamagata M, Sanes JR (2018) Expression and roles of the immunoglobulin superfamily recognition molecule sidekick1 in mouse retina. Front Mol Neurosci 11:485.

Zeng H, Sanes JR (2017) Neuronal cell-type classification: challenges, opportunities and the path forward. Nat Rev Neurosci 18:530-546.

Zhang D-Q, Zhou T-R, McMahon DG (2007) Functional heterogeneity of retinal dopaminergic neurons underlying their multiple roles in vision. J Neurosci 27:692-699.

Zheng GXY, Terry JM, Belgrader P, Ryvkin P, Bent ZW, Wilson R, Ziraldo SB, Wheeler TD, McDermott GP, Zhu J, Gregory MT, Shuga J, Montesclaros L, Underwood JG, Masquelier DA, Nishimura SY, SchnallLevin M, Wyatt PW, Hindson CM, Bharadwaj R, et al. (2017) Massively parallel digital transcriptional profiling of single cells. Nat Commun 8:14049.

Zhu YJ, Xu WW, Hauswirth SH DeVries (2014) Genetically targeted binary labeling of retinal neurons. J Neurosci 34:7845-7861. 